$7 \quad$ Ning Cheng (corresponding author)

8

9

10

11

12

13

14

14
15

15

16
17

17

18

19

20

21

22

23

24

25

26

27

28

29

30

31

32

33

34

Research Associate University of Western Australia

35 Stirling Highway, Crawley

Perth, WA 6009, Australia

Tel: +61 864883732 Fax: +61 864881044

Email: ning.cheng@research.uwa.edu.au

Mark Jason Cassidy

Australian Research Council Laureate Fellow University of Western Australia

35 Stirling Highway, Crawley

Perth, WA 6009, Australia

Tel: +61 864883732 Fax: +61 864881044

Email: mark.cassidy@uwa.edu.au

Submitted as a Paper

Words: 5544

Figures: 21

Tables:

\title{
Development of a Force-resultant Model for Spudcan Footings on Loose Sand under Combined Loads
}

\author{
Manuscript submitted to Canadian Geotechnical Journal on 30/11/2015
}

Centre for Offshore Foundation Systems and ARC CoE for Geotechnical Science and Engineering

Lloyd's Register Foundation Chair of Offshore Foundations

Centre for Offshore Foundation Systems and ARC CoE for Geotechnical Science and Engineering 


\title{
Development of a Force-resultant Model for Spudcan Footings on Loose Sand under Combined Loads
}

\author{
Ning Cheng and Mark Jason Cassidy
}

\begin{abstract}
Spudcans are typical foundations used in shallow to moderate water oil and gas fields to support jackup drilling units. Understanding the behaviour of spudcans under combined loadings is crucial to the overall response of the jack-up structure. This paper presents the development of a strain-hardening plasticity model for spudcan footing on loose sand. Most of the model components are developed from direct centrifuge observations. The centrifuge tests were performed at an acceleration of 100 times that of the Earth's gravity on a model spudcan footing subjected to combined vertical, horizontal and moment loads. All the experiments have been designed and conducted to allow the results to be interpreted with a strain-hardening plasticity framework. Combined loads were applied by using a novel apparatus, which enables independent vertical, horizontal and rotational movements of the footing. Test results also revealed the existence of a three dimensional sliding surface that intersects with the conventional yield surface. This additional surface has been defined analytically. Retrospective simulation of the experimental data using the plasticity model confirms the model's capability for the use in predicting the behaviour of larger spudcan applications offshore.
\end{abstract}

Keywords: Offshore Engineering, Centrifuge test, Force-resultant model

\section{Introduction}

Jack-ups are dynamically sensitive structures whose load response is dependent on the stiffness of their large spudcan footings. Spudcans are roughly circular in plan with a sharp protruding spigot to provide extra horizontal stability and to minimise lateral movement in the soil. Jack-ups perform drilling in water depths of up to $120 \mathrm{~m}$. At such depths, the magnitude of wind, wave and current loads acting on the hull and three legs are substantial and cause large horizontal $(H)$ and moment $(M)$ loads on the spudcan as well as changes in the vertical $(V)$. Understanding the bearing capacities of these combined $V H M$ loads is essential during the design of jack-up and during their site specific assessments (see ISO (2012)). Many studies have been conducted in the field of spudcan footings in an effort to understand their behaviour under combined VHM loadings (Noble Denton \& Associates 1987; Schotman 1989; Osborne et al. 1991; Byrne and Houlsby 1999; Gottardi et al. 1999; Martin and Houlsby 2000; Byrne and Houlsby 2001; Martin and Houlsby 2001; Cassidy et al. 2002a; Houlsby and Cassidy 2002; Cassidy et al. 2004; Bienen et al. 2006; Zhang et al. 2011). 
66 Conventional bearing capacity equations for shallow foundations were initially described by Terzaghi 67 (1943) and subsequently extended by Meyerhof (1953) and Hansen (1970). These account for 68 inclined $(V: H)$, eccentric loading $(V: M)$ and various footing shapes by using a number of 69 empirical factors applied to the bearing capacity formula for vertical loading. As such, they are not 70 suitable for the direct inclusion in numerical analyses programmes of the complete jack-up structure. 71 Recently, the offshore industry has moved towards the use of plasticity-based force-resultant models 72 to describe the behaviour of shallow foundations, as they express the footing behaviour purely in 73 terms of force resultants and can be directly coupled to typical structural analysis software used in 74 industry.

75 A number of authors have proposed such models for strip footings (Nova and Montrasio 1991; 76 Gottardi and Butterfield 1993, 1995) and circular footings (Byrne and Houlsby 1999; Gottardi et al. 77 1999; Byrne and Houlsby 2001; Cassidy et al. 2002a; Houlsby and Cassidy 2002; Bienen et al. 2006) 78 on sand. The usual approach has been to assume a yield surface, a hardening law, a flow rule and an 79 elastic region within the yield surface. An example force-resultant model for describing drained 80 behaviour of circular footing in sand is known as $\operatorname{Model} C$, a plasticity model developed by Houlsby 81 and Cassidy (2002). Model $C$ was originally based on a series of $1-g$ experimental tests performed by 82 Gottardi et al. (1999) but has also been calibrated for calcareous sand (Cassidy et al. 2002a) and 83 extended to six-degrees-of-freedom loading conditions (Bienen et al. (2006). Its use in the pushover 84 analysis of jack-ups is shown in Bienen and Cassidy (2006), Bienen and Cassidy (2009) and Cassidy 85 et al. (2010).

The four major components associated with Model $C$ are:

a) An empirical expression for the yield surface which separates the elastic and plastic boundary,

b) A model for the elastic load-displacement behaviour within the yield surface,

c) An empirical strain-hardening expression to define the expansion and contraction of the yield surface with the plastic component of vertical displacement,

d) A suitable flow rule to allow for the prediction of the footing plastic displacements during yield.

93 Further reference to Model $C$ can be found in Houlsby and Cassidy (2002), Cassidy et al. (2002a), 94 Byrne and Houlsby $(1999,2001)$ and Bienen et al. (2006). Although there is considerable 95 experimental evidence to support those strain-hardening plasticity models, most of the components 96 were developed from small-scale model 1-g experiments. This paper addresses if these accurately 97 replicate the soil-stress strain relationship of the much larger prototype spudcan footing. 
In an attempt to ensure confidence when employing Model $C$ in larger footings applications, Cassidy (2007) verified components of Model $C$ with centrifuge experiments on a flat circular footing. Although, a similar yield surface shape was broadly observed, a complete $V H M$ yield surface could not be tested because only a fixed arm (pure horizontal translation) or a hinged arm (pure rotation) were used (see details of the apparatus in Cassidy et al. (2004)). Subsequently, Bienen et al. (2007) conducted a series of centrifuge tests, also on a shallow circular foundation on sand, but in this case only in the vertical-torsional loading plane. Following the same theoretical framework of strainhardening plasticity, Govoni et al. $(2010,2011)$ investigated circular footings resting on medium dense silica sand subjected to combined vertical, moment and horizontal loading and compared results to existing data obtained at $1-g$. Special emphasis was given to the embedment effect. However, only two specific load paths were investigated, as the same fixed and hinged arms were used in this experiment as in Cassidy (2007). Cocjin and Kusakabe (2012) conducted a series of displacement controlled vertical loading and swipe tests at various vertical load levels on a flat strip footing. The study confirms that the deviatoric load capacity is highly dependent on the ratio of the vertical load and vertical peak bearing capacity. However, only horizontal swipe tests were performed in their study.

Although there is considerable experimental evidence from the small scale 1- $g$ environment to support the well-established strain-hardening plasticity models, no comprehensive centrifuge evidence, to date, has been provided. This is because with only single or at the best two load paths, it is difficult to establish any complete yield surface or flow rule in three dimensional space. Another restriction of the previous studies is that the small-scale model being tested was a representative flat circular plate; no model with an inverted conical spudcan shape has been studied. A summary of the major studies in the framework of plasticity based force-resultant models is provided in Table 1.

Table 1 Comparison of the major studies publicly available concerning plasticity based force-resultant models

\begin{tabular}{|c|c|c|c|c|c|c|c|c|}
\hline & \multicolumn{2}{|c|}{ Footing type } & \multirow[t]{2}{*}{ Sand type } & \multirow{2}{*}{$\begin{array}{c}\mathrm{g} \\
\text { level }\end{array}$} & \multirow{2}{*}{$\begin{array}{l}V H M \\
\text { load } \\
\text { paths }\end{array}$} & \multirow{2}{*}{$\begin{array}{l}\text { Yield } \\
\text { Surface }\end{array}$} & \multirow{2}{*}{$\begin{array}{c}\text { Flow } \\
\text { rule }\end{array}$} & \multirow{2}{*}{$\begin{array}{c}\text { Force- } \\
\text { resultant } \\
\text { model }\end{array}$} \\
\hline & $\begin{array}{c}\text { Flat } \\
\text { circular }\end{array}$ & Spudcan & & & & & & \\
\hline Gottardi (1999) & $\sqrt{ }$ & & $\begin{array}{l}\text { Very } \\
\text { dense }\end{array}$ & 1 & Full & $\sqrt{ }$ & $\sqrt{ }$ & \\
\hline Byre \& Houlsby (1999) & $\sqrt{ }$ & & $\begin{array}{l}\text { Very } \\
\text { dense }\end{array}$ & 1 & Full & $\sqrt{ }$ & & \\
\hline $\begin{array}{l}\text { Byrne \& Houlsby } \\
\text { (2001) }\end{array}$ & $\sqrt{ }$ & & Carbonate & 1 & Full & $\sqrt{ }$ & & \\
\hline $\begin{array}{l}\text { Houlsby \& Cassidy } \\
\qquad(2002)\end{array}$ & $\sqrt{ }$ & & $\begin{array}{l}\text { Very } \\
\text { dense }\end{array}$ & 1 & Full & $\sqrt{ }$ & $\sqrt{ }$ & $\sqrt{ }$ \\
\hline Cassidy (2002) & $\sqrt{ }$ & & Carbonate & 1 & Full & $\sqrt{ }$ & $\sqrt{ }$ & $\checkmark$ \\
\hline Bienen (2006) & $\sqrt{ }$ & & Loose & 1 & Full & $\sqrt{ }$ & $\sqrt{ }$ & $\sqrt{ }$ \\
\hline Cassidy (2007) & $\checkmark$ & & Loose & 100 & 2 & & & \\
\hline Govani $(2010,2011)$ & $\sqrt{ }$ & & Medium & 100 & 2 & & & \\
\hline $\begin{array}{c}\text { Cojin \& Kusakabe } \\
\text { (2012) }\end{array}$ & $\sqrt{ }$ & & Dense & 50 & 1 & & & \\
\hline
\end{tabular}




\begin{tabular}{llllllll}
\hline This study & $\checkmark$ & Loose & 100 & Full & $\checkmark$ & $\checkmark$ & $\sqrt{ }$ \\
\hline
\end{tabular}

22 This paper details such a plasticity-based force-resultant model for spudcan footing on loose sand

124

125

126

127

128

with most of the model components developed from direct centrifuge observations. The experiments were conducted at an acceleration of 100 times that of the Earth's gravity in the drum centrifuge at the University of Western Australia (UWA) and is the first comprehensive centrifuge programme investigating spudcan behaviours on sand in all VHM load directions.

\section{Experimental setup}

The sign convention for the loads and displacements adopted in this study follow the standardised convention system proposed by Butterfield et al. (1997) for combined loadings. Foundational loading was applied by means of displacement-control to a load reference point (LRP). The LRP is located at the centreline of the foundation at the mud line level. The load was described by horizontal translation $(u)$, vertical translation $(w)$ and rotation $(\theta)$; positive sign conventions together with size dimensions are shown in Fig. 1.

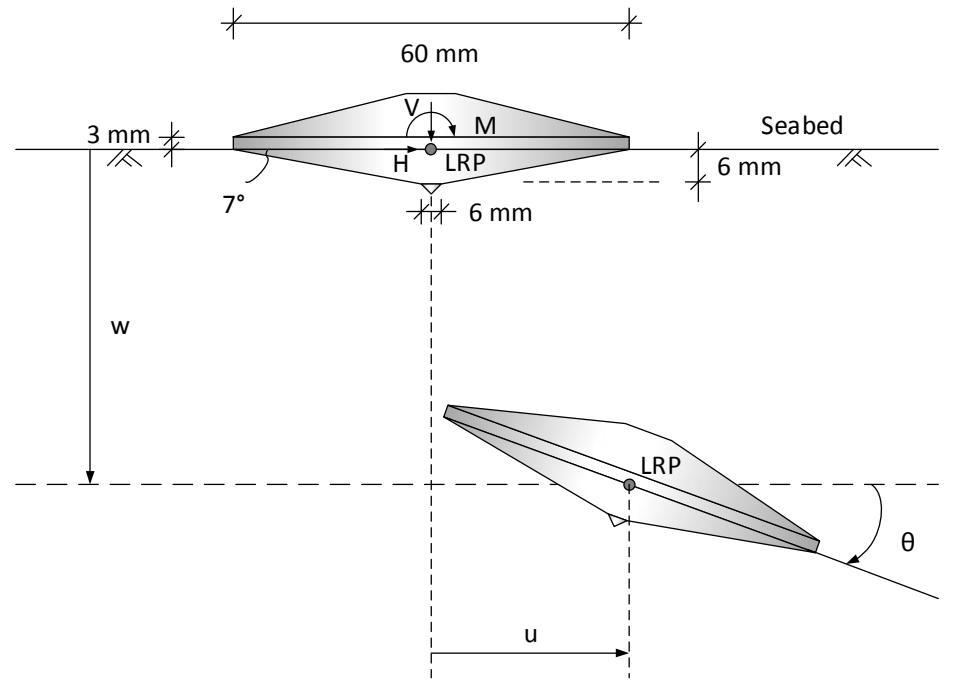

Fig. 1 Model dimensions and sign conventions for load and displacement

The experiments were conducted at the UWA drum centrifuge, which has a swinging platform at a radius of $0.6 \mathrm{~m}$ and a maximum rotational speed of $850 \mathrm{rev} / \mathrm{min}$. The drum centrifuge consists of a central tool table, driven by a Dynaserv servo motor, and a channel for sample placement, which are able to rotate differentially. This creates relative motion between the sample and any tool connected to the actuator, situated within the tool table. The soil channel containment area has width of $300 \mathrm{~mm}$ and a depth of $200 \mathrm{~mm}$. Details of this drum centrifuge are provided by Stewart et al. (1998).

A recently developed VHM apparatus (Zhang et al. 2013) was used in the experiments. The conventional drum centrifuge configuration only allowed for a setup direction perpendicular to the 
144

145

146

147

148

149

150

151

152

153

154

155

156

vertical and horizontal plane on the footing ( $w$ and $u$, respectively), thus only two degree-of-freedom movement was available, as shown in Fig. 2. A third degree-of-freedom movement was achieved, by adding another actuator on top of the existing actuator and connected through linkage arms and hinges (Fig. 3(c)). This enables the footing to also rotate in-plane.

Soil channel

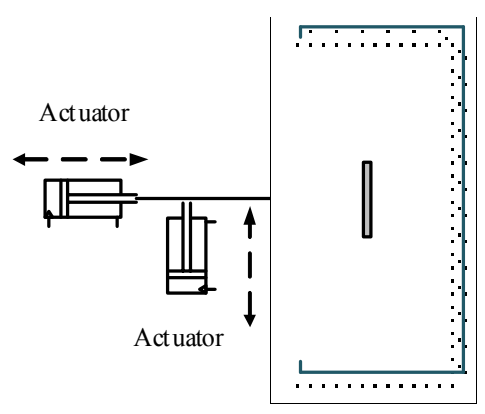

Fig. 2 Schematic diagram for conventional actuators in drum centrifuge

The primary advantage of using this displacement controlled apparatus is the ability to apply any combination of the displacement paths to the model footing using computer controlled actuators. This series of movements were calculated in real time by a build-in-house programme developed with NI LabVIEW interface (National Instrument Corporation 2003). More details of the apparatus used in this experiment can be found in Zhang et al. (2013), Cheng et al. (2014) and Cheng and Cassidy (2016). Photos showing the VHM apparatus incorporated in the drum centrifuge are presented in Fig. 3(a) and (b).

(a)

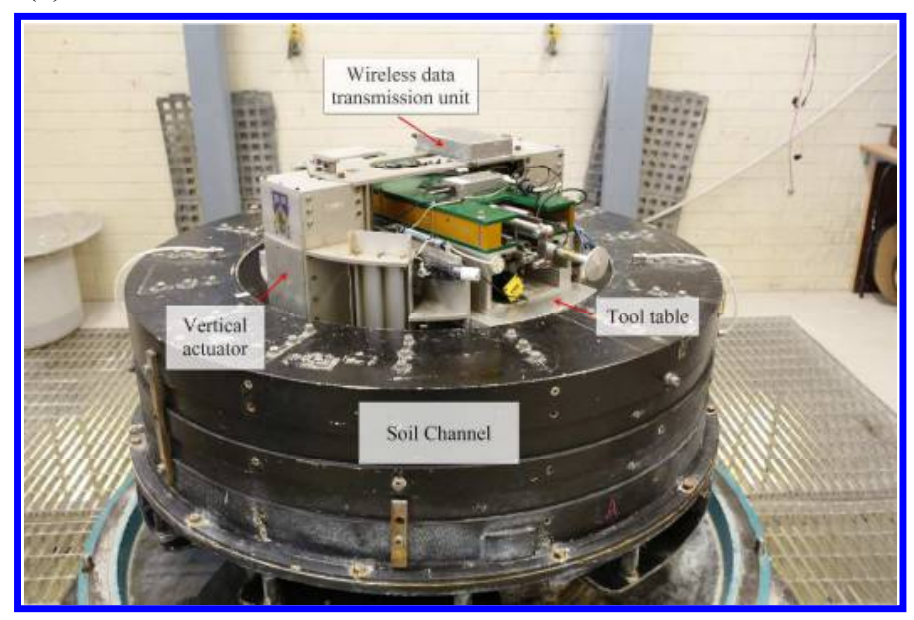

157

158

Fig. 3 Picture and schematic drawing of the $V H M$ loading arm

Important features of the new VHM apparatus are: (b)

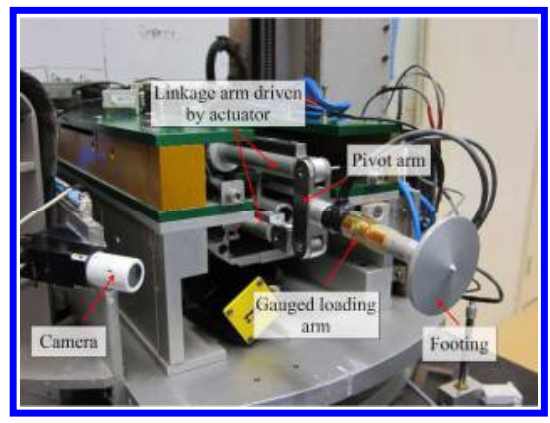

(c)

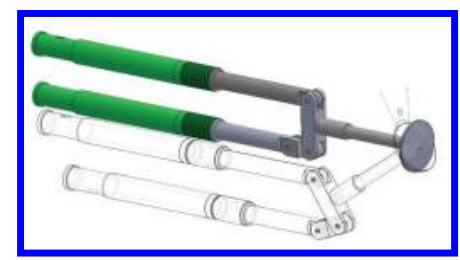


a) The new $V H M$ apparatus can conduct any specified combination of vertical, horizontal and rotational movement with speeds ranging from $0.1 \mathrm{~mm} / \mathrm{s}$ to $3 \mathrm{~mm} / \mathrm{s}$.

b) The new VHM apparatus has been equipped with modern inflight computers to ensure the highest possible accuracy along with the minimum systematic lag. This is particularly important for tests involving sequential movements, such as the swipe tests described in this paper.

c) The new VHM apparatus has been retrofitted with enhanced mechanical systems. This ensures the apparatus can be safely used in high stress testing environment so it is adaptable to any soil.

\section{Soil characterisation}

The soil used in this study was a superfine silica sand used in numerous UWA studies. The physical properties of the sand are listed in Table 2 (Cassidy and Cheong 2005).

Table 2. Soil properties of sand used in test

\begin{tabular}{|l|l|}
\hline Dry unit weight $\left(\mathrm{kN} / \mathrm{m}^{3}\right)$ & \\
Particle size, $D_{50}, D_{10}, D_{20}: \mathrm{mm}$ & $0.19,0.099,0.135$ \\
Minimum dry density, $\rho_{\min }: \mathrm{kg} / \mathrm{m}^{3}$ & 1517 \\
Maximum dry density, $\rho_{\max }: \mathrm{kg} / \mathrm{m}^{3}$ & 1829 \\
Maximum $e_{\max }$ & 0.75 \\
Minimum $e_{\min }$ & 0.53 \\
Specific gravity, $G_{S}$ & 2.65 \\
Critical-state frictional angle: degrees & 31 \\
\hline
\end{tabular}

The sand sample was prepared using a rotating actuator to rain the silica sand into the rotating outer channel of the drum centrifuge. The sample height was $140 \mathrm{~mm}$ and remained saturated with $20 \mathrm{~mm}$ water above the surface throughout the test. A consistent relative density of 30-35\% was interpreted from 8 cone penetrometer tests (Fig. 4) using the procedure of Schneider and Lehane (2006) where

$R_{d}=100 \sqrt{\frac{Q}{250}}$ and where $Q=\left(q_{c}-\sigma_{V}\right) / \sigma_{V}^{\prime}, q_{c}$ is the cone tip resistance, $\sigma_{V}$ and $\sigma_{V}^{\prime}$ are the current total and effective stress levels respectively. 


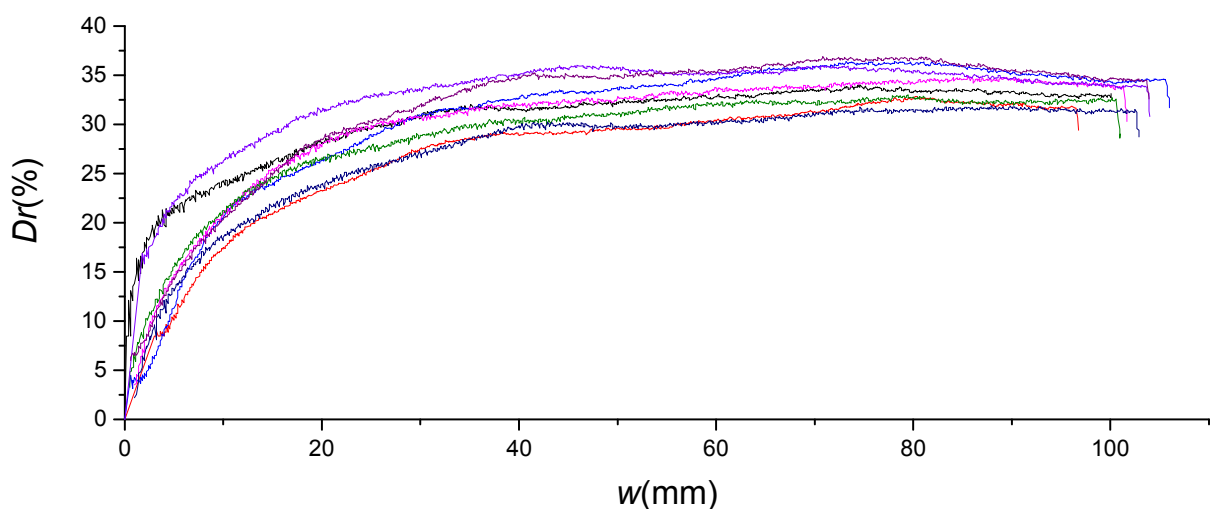

180

181

182 Experimental plan

183 Five distinct types of footing tests were conducted in the experimental programme: vertical 184 penetration, vertical load-unload loops, swipe, monotonic radial displacement test and combined 185

Fig. 4 Interpretation of cone penetrometer test results radial displacement test. The vertical penetration tests were used to study the variation of the size of the yield surface, $V_{0}$, with spudcan penetration and the load-unload tests the relevant elastic behaviour. Tan (1990) introduced the swipe test for a study of conical footings on sand under combined loadings. In the swipe tests of this study, the footing was vertically penetrated to a prescribed depth where the targeted stress level was achieved $(\approx 350 \mathrm{kPa})$. Then, the footing was subjected to a series of combined horizontal displacement and rotational excursions. Radial displacement tests were used to investigate the flow rule, in which the footing was subjected to different combinations of vertical and horizontal displacements or vertical and rotational displacements. It has to be noted that due to the limited testing space, in the paper no yield surface variation with $V_{0}$ has been investigated. The detailed experimental plan is listed in Table 3. Vertical loading tests and swipe tests were already presented in Cheng and Cassidy (2016), where a comprehensive interpretation is also given in terms of the determination of the yield surface and additional sliding surface. This paper is mainly relevant to the development of force-resultant model, presentation of radial displacement tests, calibration of the flow rule and model assessment through retrospective analysis of selected experimental data. 


\begin{tabular}{|c|c|c|c|c|c|c|c|c|}
\hline Test type & $\begin{array}{c}\text { Test } \\
\text { name }\end{array}$ & $V(\mathrm{~N})$ & $\begin{array}{l}V / A \\
(\mathrm{kPa})\end{array}$ & $u / 2 R \theta$ & $\begin{array}{c}w \\
(\mathrm{~mm})\end{array}$ & $\begin{array}{c}u \\
(\mathrm{~mm})\end{array}$ & $\begin{array}{c}\theta \\
\text { (Radians) }\end{array}$ & Figures \\
\hline $\begin{array}{c}\text { Vertical } \\
\text { penetration }\end{array}$ & SVP1 & 4434 & 1568 & - & 25.8 & - & - & Fig.5 \\
\hline $\begin{array}{l}\text { Load-unload } \\
\text { loops }\end{array}$ & SVP2 & 3810 & 1348 & - & 19.8 & - & - & Fig.5 \\
\hline \multirow[t]{7}{*}{ Swipe tests ${ }^{1}$} & SW1 & 1287 & 455 & $\infty$ & & 9 & 0 & \multirow[t]{7}{*}{ Fig. 6-11 } \\
\hline & SW2 & 966 & 342 & 0.531 & & 5 & 0.157 & \\
\hline & SW3 & 1150 & 407 & 0.095 & & 0.9 & 0.157 & \\
\hline & SW4 & 903 & 319 & -0.095 & & -0.9 & 0.157 & \\
\hline & SW5 & 1047 & 370 & -0.286 & & -2.7 & 0.157 & \\
\hline & SW6 & 1035 & 366 & -0.572 & & -5.4 & 0.157 & \\
\hline & SW7 & 1197 & 423 & -1.145 & & -9 & 0.131 & \\
\hline Monotonic & RD1 & 1822 & 644 & - & 15 & 15 & - & Fig. 14,15 \\
\hline Radial & RD2 & 2385 & 843 & - & 15 & 4.5 & - & \\
\hline \multirow{4}{*}{$\begin{array}{l}\text { displacement } \\
\text { tests }\end{array}$} & RD3 & 2152 & 761 & - & 15 & 9 & - & \\
\hline & RD4 & 1654 & 585 & - & 8.5 & - & 0.148 & \\
\hline & RD5 & 748 & 265 & - & 4.5 & - & 0.157 & \\
\hline & RD6 & 3240 & 1146 & - & 17.7 & - & 0.154 & \\
\hline \multirow{2}{*}{$\begin{array}{l}\text { Combined } \\
\text { radial } \\
\text { displacement } \\
\text { tests }\end{array}$} & RD7 & 1128 & 399 & 0.102 & 9 & 9 & 0.157 & \multirow[t]{2}{*}{ Fig. 14,15} \\
\hline & RD8 & 1793 & 634 & 0.971 & 12 & 6 & 0.105 & \\
\hline
\end{tabular}

\section{Results and discussion}

203 In the following section, the results from the experiments are described with respect to the four major components required for the strain-hardening plasticity model.

Hardening law

The strain-hardening expression, known as the hardening law, is used to define the variation of the size of the yield surface. For most shallow foundation models, the size of the yield surface is reasonably deemed as a function of the plastic component of the vertical displacement (Gottardi et al. 1999; Byrne and Houlsby 2001; Cassidy et al. 2002a; Houlsby and Cassidy 2002). One of the key assumption of the strain-hardening plasticity theory is that when the footing is pushed into soil, the shape of the yield surface is assumed constant; however, the size expands with the amount of plastic vertical displacement $\left(w_{p}\right)$. Shown in Fig. 5 are the vertical loading response of two vertical loading tests SVP1 and SVP2 which show consistency in the experimental programme. From the unloadreload loops of SVP2 an average elastic stiffness $K_{V}$ was established as $2.4 \mathrm{kN} / \mathrm{mm}$. The stiffness of the loading system was taken into consideration through a correction process. In this process, additional plastic penetration can be calculated for a given change in vertical load and measured 
217 actual vertical displacement. If the form of the hardening law is known (such as Equation (2)), then a 218 correction can be applied to update $V_{0}$ from its value at the beginning of the test.

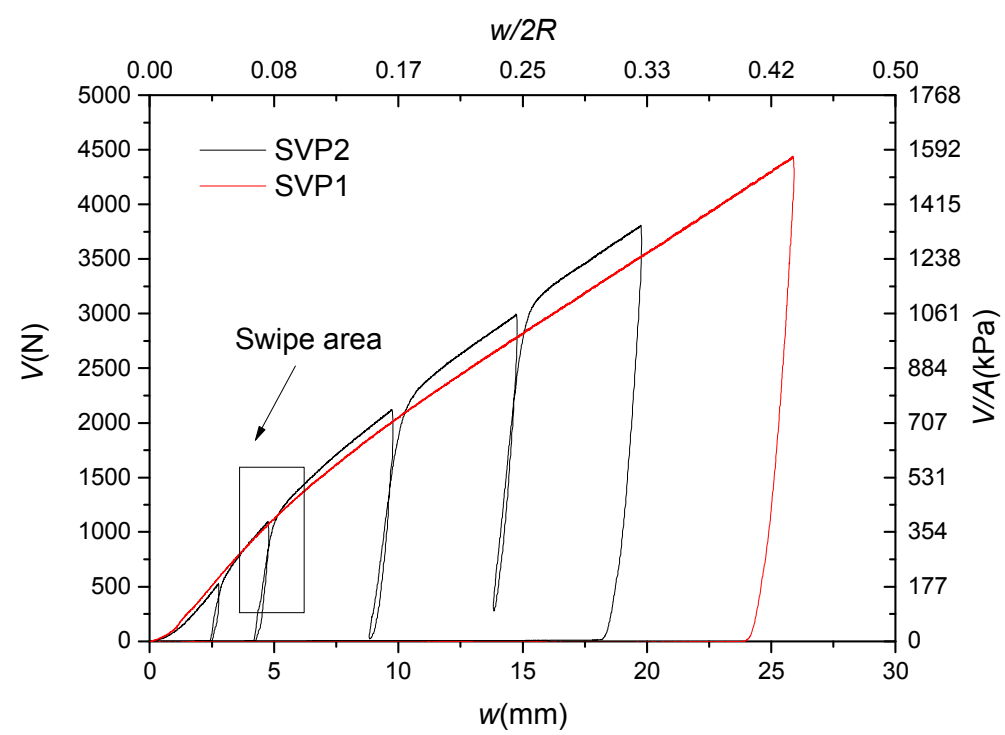

219

220 Fig. 5 Vertical penetration and load-unload loops

221 An empirical strain-hardening expression is used to define the expansion and contraction of the yield 222 surface with the plastic component of vertical displacement. With the elastic vertical displacements 223 removed, a function previously used to fit carbonate sand experimental data (Cassidy et al. 2002a) 224 was again used here to fit a hardening law:

$225 \quad V_{0}=\frac{c w_{p}+k_{2} w_{p}^{2}}{k_{1}+w_{p}}$

226 The best fit model parameters are found to be $c=278 \mathrm{~N}, k_{1}=0.15 \mathrm{~mm}, k_{2}=0.04 \mathrm{~N} / \mathrm{mm}$. No special 227 significance is attached to this equation except that it represents a good fit to the vertical loadpenetration response. Alternative expressions could also be appropriate.

Elastic stiffness

230 Increments within the yield surface are assumed to be elastic and follow:

$\left\{\begin{array}{c}d V \\ d M / 2 R \\ d H\end{array}\right\}=2 G R\left[\begin{array}{ccc}k_{V} & 0 & 0 \\ 0 & k_{M} & k_{C} \\ 0 & k_{C} & k_{H}\end{array}\right]\left\{\begin{array}{c}d w \\ 2 R d \theta \\ d u\end{array}\right\}$

where $G$ is a representative elastic shear modulus and $k_{V}, k_{M}, k_{H}, k_{C}$ are dimensionless elastic constants for elastic behaviour within the yield surface, and $(d V, d M, d H)$ and $(d w, d \theta, d u)$ 
234 vectors of incremental load and displacement respectively. The elastic coefficients have been defined 235 using finite element methods by Bell (1991), Ngo-Tran (1996) and Doherty and Deeks (2003), and 236 appropriate values for circular foundations, as listed in Doherty and Deeks (2003) are:

$237 \quad k_{V}=2.904 ; k_{M}=0.548 ; k_{H}=2.901 ; k_{C}=-0.208$.

238 The shear modulus $G$ was estimated by the following formula, suggested by Houlsby and Cassidy 239 (2002):

$240 \quad \frac{G}{p_{a}}=g \sqrt{\frac{V}{A p_{a}}}$

241 where $p_{a}$ is atmospheric pressure, $V$ is a representative vertical load on the foundation, $A$ is the plan 242 area of the foundation and $g$ is a dimensionless constant. This latter value of $g$ can be back calculated 243 from the unload-reload test SVP2 as 44.2 (representing an average $G$ of $13.8 \mathrm{~N} / \mathrm{mm}^{2}$ and $k_{V}=2.904$ ). 244 Without measured data of the stiffness Cassidy et al. (2002b) recommend a $g$ value of approximately 245400 for practical offshore conditions.

246 Yield surface

247 Following Tan (1990) and Martin and Houlsby (2000), swipe tests were used to explore the yield 248 surface shape. Fig. 6 shows the recorded load path of swipe test SW2 with $M / 2 R: H \approx 1.5$ in three249 dimensional space and its projected view in corresponding the $V: H, V: M / 2 R$ and $H: M / 2 R$ 250 planes. The colour bar in Fig. 6(a) represents the $M / 2 R: H$ ratio on each test point; as shown in the 251 figure, despite some changes occurring at the initial stage, the $M / 2 R H$ ratio is consistent throughout 252 the test. Another interesting trend that can be observed from Fig. 6 (d) is the sliding failure 253 mechanism occurred at the $V / V_{0} \approx 0.25$ where the horizontal force continued to increase while 254 moment load kept constant. This behaviour was not observed from previous centrifuge studies when 255 only the horizontal displacement path was applied. 

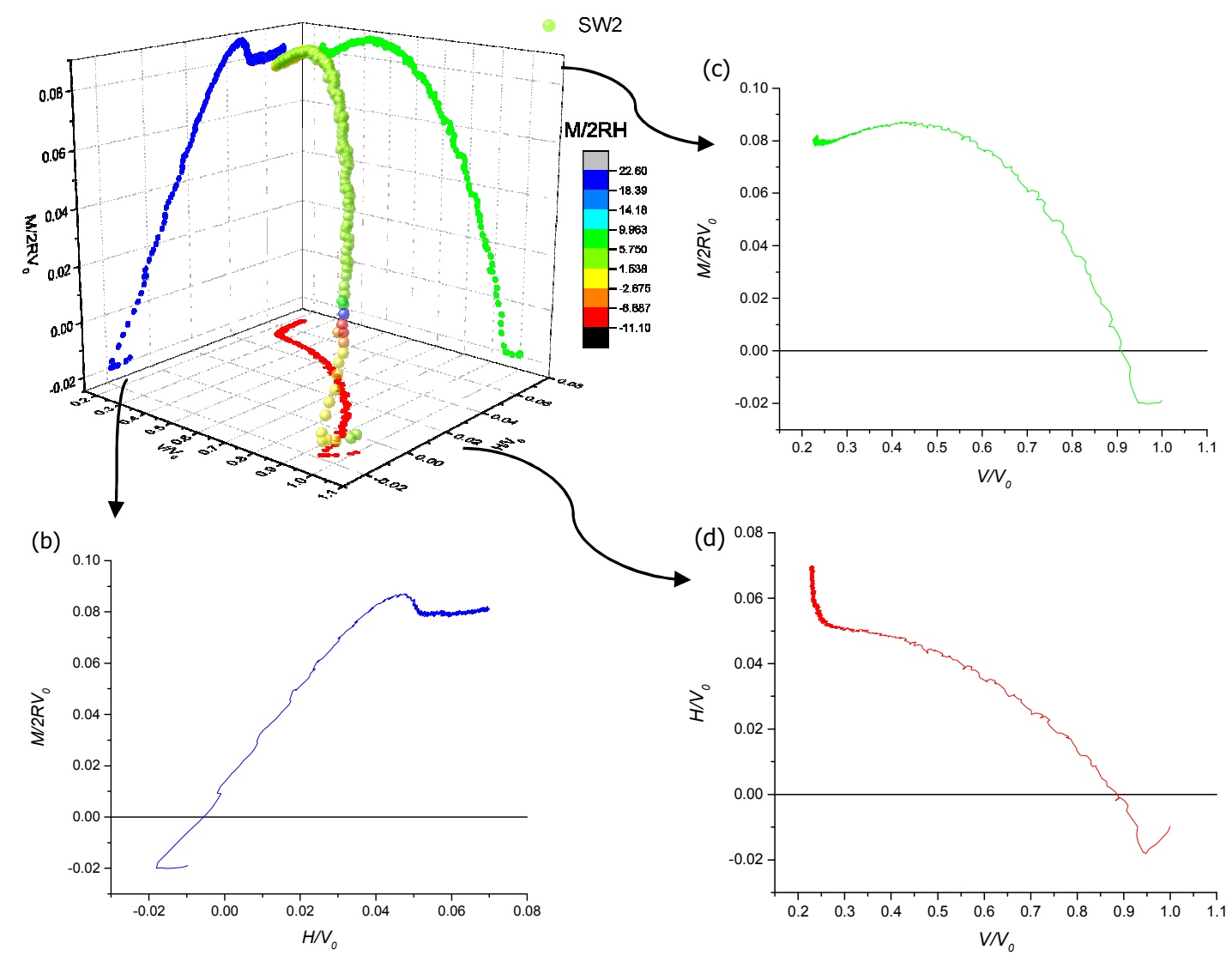

Fig. 6 Swipe test SW2 in three-dimensional and corresponding projected views

258 Each swipe test follows one particular $M / 2 R H$ ratio corresponding to one specific load path on the yield surface. Fig. 7 presents all swipe tests collapsed into one three-dimensional space. It can be observed that as moment increases (from SW1 to SW7), the sliding failure mechanism becomes more pronounced. Cassidy (2007) reported that a critical state occurred at $V / V_{0} \approx 0.3$. Clearly, this is not supported in the current study, as the critical state point occurs at different $V / V_{0}$ ratios for different moment to horizontal load ratios. This is demonstrated in Fig. 7. Due to the three-dimensional nature of the sliding surface that intersects with the yield surface the location of the transition point cannot be determined solely on the $V / V_{0}$ ratio but also depending on the ratio of moment to horizontal load. 


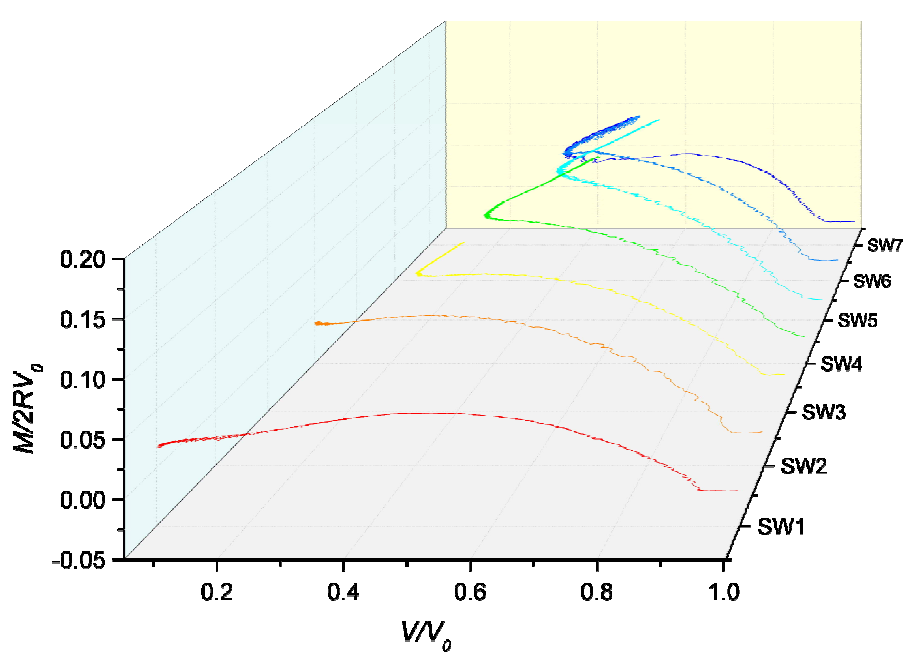

268 Fig. 7 All swipe tests in $V / V_{0}: M / 2 R V_{0}$ space
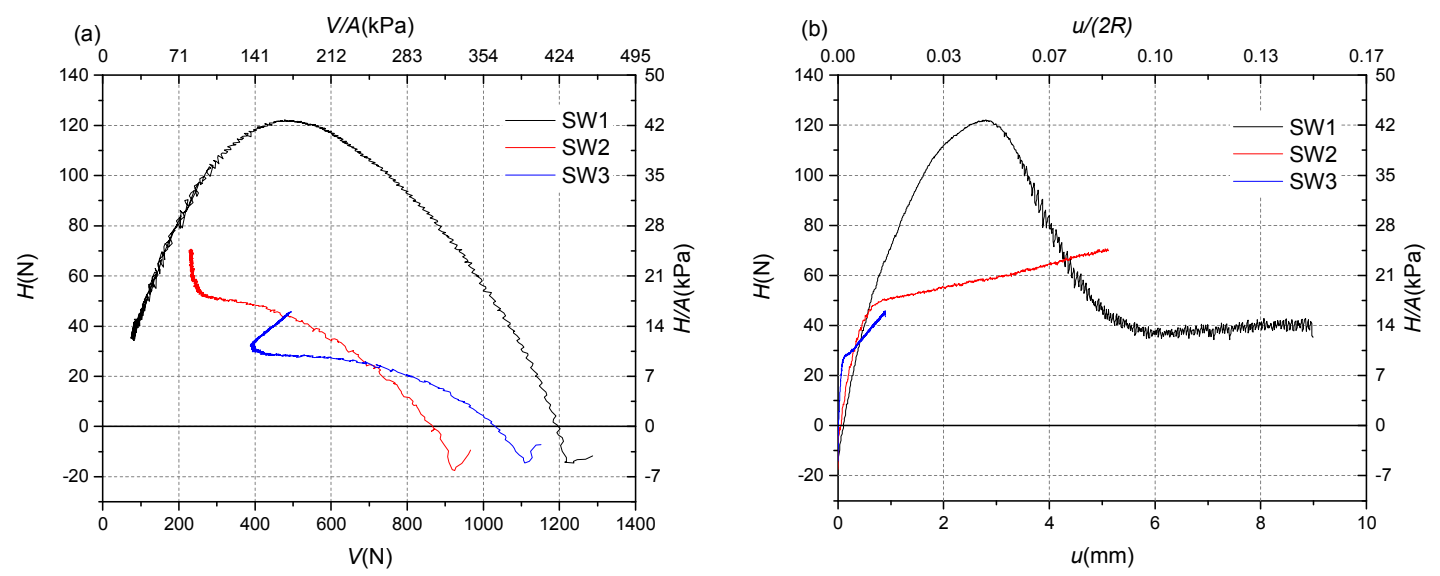

269

Fig. 8 Representative swipe tests projected on the $V: H$ and $H: u$ plane

270 Fig. 8 shows three representative swipe tests projected on the $V: H$ plane and their corresponding load-displacement curves. SW1 is a conventional, pure translation test, which can be observed from most previous studies. It should be noted that the $M / 2 R: H$ ratio increases from test SW1 to test SW3. In SW2 and SW3, after a certain point, $V$ starts to increase rather than decrease as in test SW1. This transition point varies depending on the $M / 2 R: H$ ratios which means both the horizontal load and the moment load play an important role in determining this point. Gottardi et al. (1999) and Byrne and Houlsby (2001) derived a three-dimensional surface from the experimental swipe tests in a $1 \mathrm{~g}$ environment. This surface could be defined as

$$
f=\left(\frac{H}{h_{0} V_{0}}\right)^{2}+\left(\frac{M / 2 \mathrm{R}}{m_{0} V_{0}}\right)^{2}-\frac{2 \alpha H M / 2 R}{h_{0} m_{0} V_{0}^{2}}-\left[\frac{\left(\beta_{1}+\beta_{2}\right)^{\left(\beta_{1}+\beta_{2}\right)}}{\beta_{1}^{\beta_{1}} \beta_{2}^{\beta_{2}}}\right]^{2}\left(\frac{V}{V_{0}}\right)^{2 \beta_{1}}\left(1-\frac{V}{V_{0}}\right)^{2 \beta_{2}}=0
$$


279 where $V_{0}$ denoted reference vertical load and representing the apex of the yield surface determined by 280 the hardening law such as Equation (1). Parameters $\mathrm{h}_{0}$ and $\mathrm{m}_{0}$ define the extent of the yield surface in 281 the moment and horizontal directions respectively. The shape factors $\alpha$ effects the eccentricity of the 282 elliptical cross-section of the yield envelope in the $H: M / 2 R$ plane. The factors $\beta_{1}$ and $\beta_{2}$ determine 283 the position of the maximum size of the elliptical section between $V=\beta_{2} V_{0} /\left(\beta_{1}+\beta_{2}\right)$ and $V=V_{0} / 2$ 284 (Nova and Montrasio 1991). Previous studies have established various sets of parameters for flat 285 circular footing on sand. For comparison, those parameters are listed in Table 4 along with the 286 parameters identified in the current study (through linear least squares fitting).

Table 4. Comparison of yield surface shape parameters

\begin{tabular}{c|cccc}
\hline & $\begin{array}{c}\text { Byrne and } \\
\text { Houlsby (2001) }\end{array}$ & $\begin{array}{c}\text { Houlsby and } \\
\text { Cassidy (2002) }\end{array}$ & $\begin{array}{c}\text { Bienen et al. } \\
(2006)\end{array}$ & This study \\
\hline$h_{0}$ & 0.154 & 0.116 & 0.122 & 0.113 \\
$m_{0}$ & 0.094 & 0.086 & 0.094 & 0.096 \\
$\alpha$ & -0.25 & -0.2 & -0.25 & -0.248 \\
$\beta_{1}$ & 0.82 & 0.90 & 0.76 & 0.71 \\
$\beta_{2}$ & 0.82 & 0.99 & 0.76 & 0.99 \\
Sand & Carbonate & Very dense & Very loose & Loose \\
type & & & & 100 \\
glevel & 1 & 1 & 1 & \\
\hline
\end{tabular}

288 Fig. 9 shows a great consistency of these parameters when compared to the experimental data and the 289 proposed yield surface normalised in the $H: M / 2 R$ plane. 


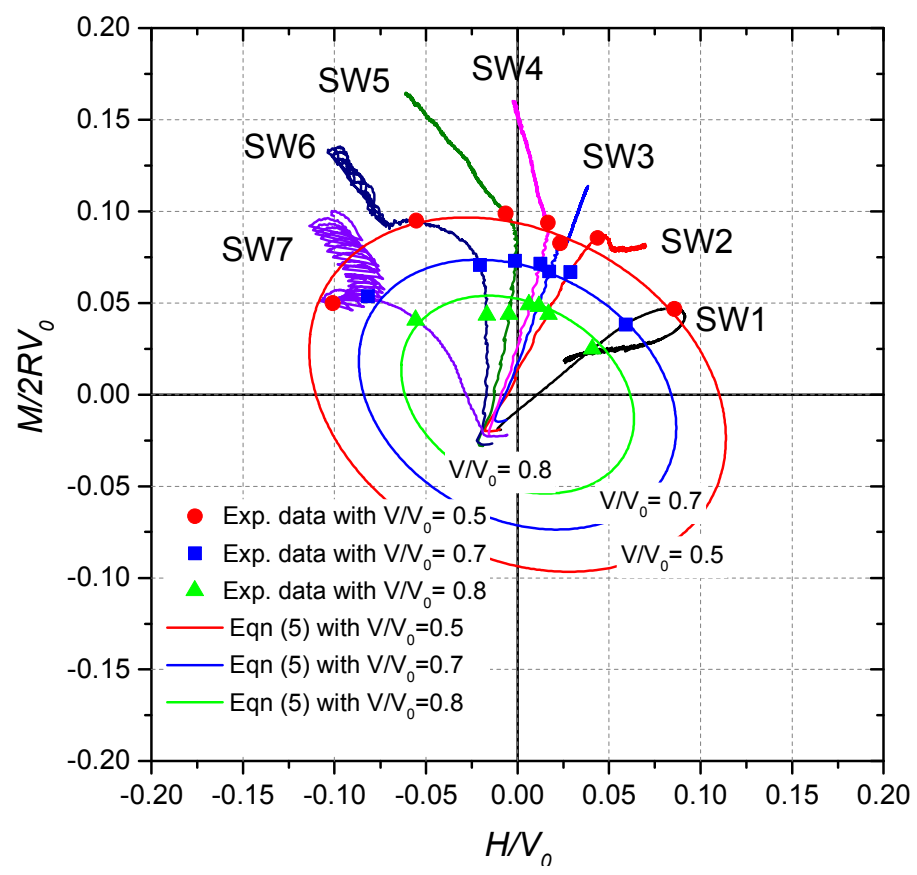

292 Fig. 9 Comparison between the analytical $\mathrm{VHM}$ yield surface and the experimental data for swipe tests

293 The overall quality of the fitting was defined by introducing the quantity

294

$Q^{2}=\left(\frac{H}{h_{0}}\right)^{2}+\left(\frac{M / 2 R}{m_{0}}\right)^{2}-\frac{2 \alpha H M / 2 R}{h_{0} m_{0}}$

295 Fig. 10 shows the load paths of all swipe tests projected onto the normalised $V: Q$ plane, validating the 296 best-fit parameters previously mentioned. It has to be noted that all the load paths that deviate from 297 the yield surface has been removed in this figure for data fitting purpose as Equation (6) was not 298 intended for describing sliding phenomena. 


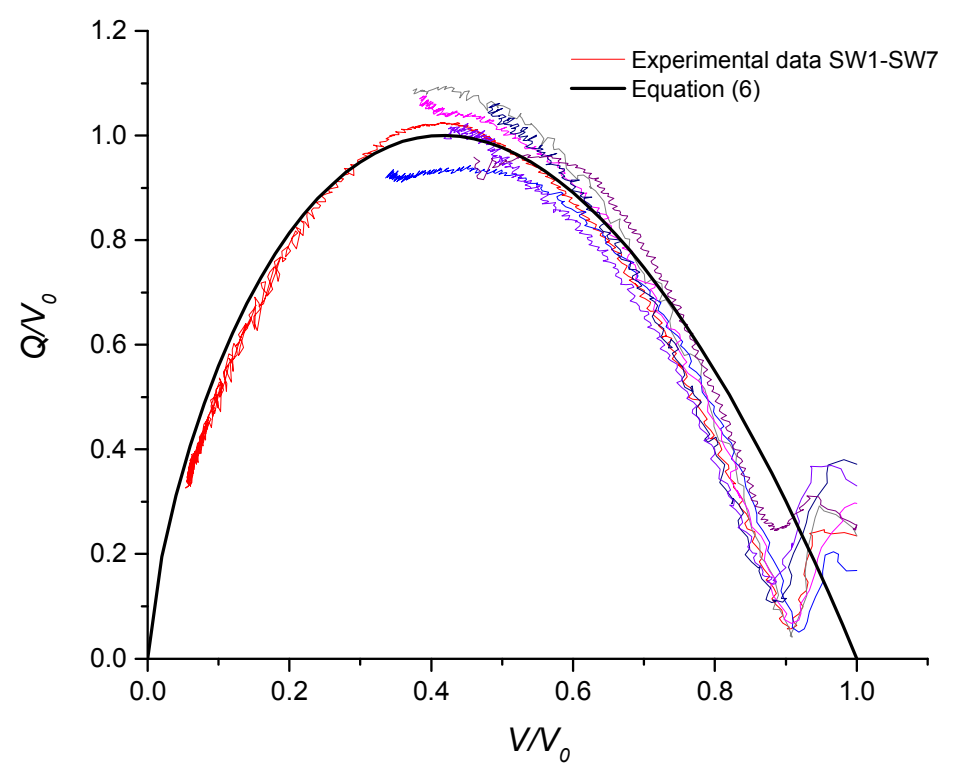

299

300

301

302

Fig. 10 Swipe load path against Equation (6) in the normalised $H: M$ plane

When all swipe tests are plot in three-dimensional space, it becomes clear that the load paths that diverge from yield surface lie on one surface. This surface could be described in the form of

$$
h=\frac{1}{\tan \phi}\left(\frac{H}{V_{0}}\right)-4\left(\frac{M / 2 R}{V_{0}}\right)+\frac{V}{V_{0}}=0
$$

by assuming a simple Coulomb failure criterion for pure shearing occurred beneath the footing. More details of the formulation are provided in Cheng and Cassidy (2016). The mobilised internal friction angle $\phi$ during the centrifuge tests was determined from a iterative procedure developed by White et al. (2008). In this study, $\phi$ was estimated to be $34.3^{\circ}$ (see, Cheng and Cassidy (2016)). With $\phi$ determined, the theoretical sliding surface is defined. Fig. 11 demonstrates the proposed complete form of the yield surface supplemented with the sliding surface in three-dimensional space. This three-dimensional sliding surface intersects the conventional yield surface with certain angles: in $V: H$ plane this angle is determined by mobilised internal friction angles $\tan \phi$ while in $V:$ M plane this angle is of a fixed value. Further, the intersection lines between the yield surface and sliding surface clearly defines all the transition points. 


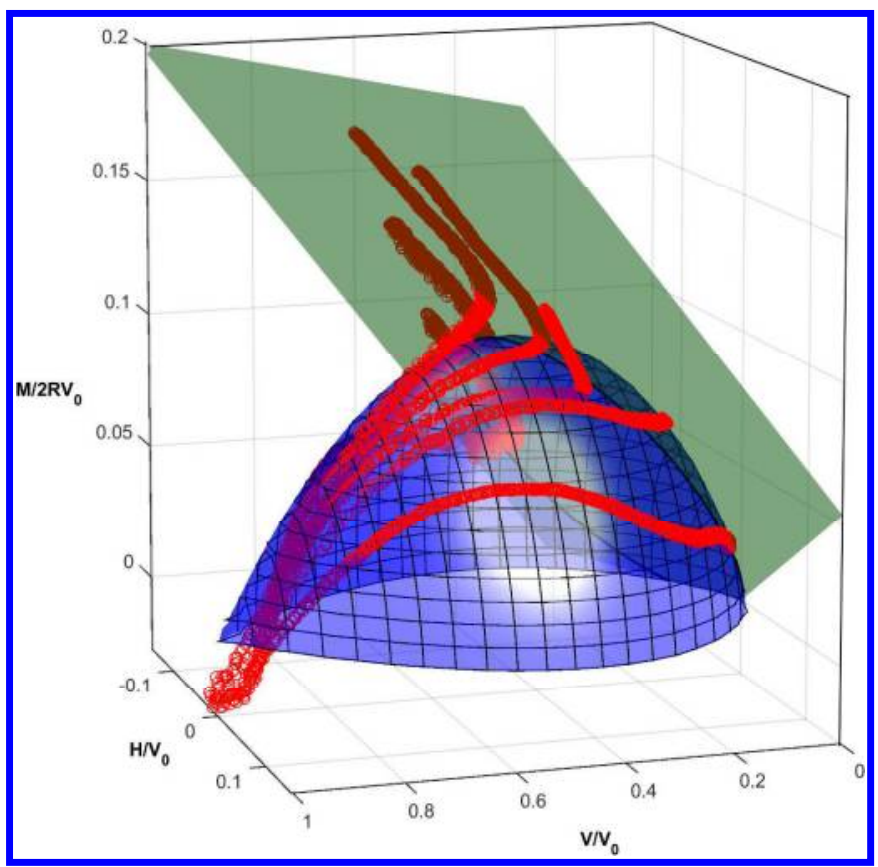

314 Fig. 11 Yield surface intersected with sliding surface in three dimensional space

315 In order to evaluate the performance of this integrated yield-sliding surface model, the proposed yield surface along with the sliding surface were sliced cut with the $M / 2 R H$ ratio taken from the experiments then projected to $V: H$ and $V: M$ planes respectively in two dimensions.

Fig. 12 shows a great consistency when compare the experimental measured value of $V / V_{0}$ at the parallel and transition point and the corresponding $M / 2 R H$ ratio against those values predicted from the theoretical yield surface (Equation (5)) and sliding surface (Equation (7)). The solid blackline represents the model predictions with values greater than 0 while the redline represents those values less than 0. Both lines were derived by solving Equation (5) and (7) simultaneously.

Fig. 13 shows the comparison between experimental measured swipe tests SW3 with the theoretical yield surfaces proposed in previous studies along with the sliding surface derived above. Equation (5) with the parameters derived in this study provides the best fit. Bienen et al. (2012) parameters slightly under estimate both the horizontal and moment load capacities, while the parameters of Byrne and Houlsby (2001) failed to exactly locate the transition point between surfaces of Equation (5) and (7). It is interesting to note that after the transition point, the consecutive load path tracks along the sliding surface resulting in an increase in vertical load. Cassidy (2007) also reported the existence of such a transition point occurred at $V / V_{0} \approx 0.3$ for flat circular foundation. However, it has to be emphasised that, this transition point can vary from $V / V_{0} \approx 0.45$ to $V / V_{0} \approx 0.05$, depending on the ratio of moment to horizontal load due to the three-dimensional nature of the sliding surface. This is explained with the data of this paper and the fit of Equation (5). 


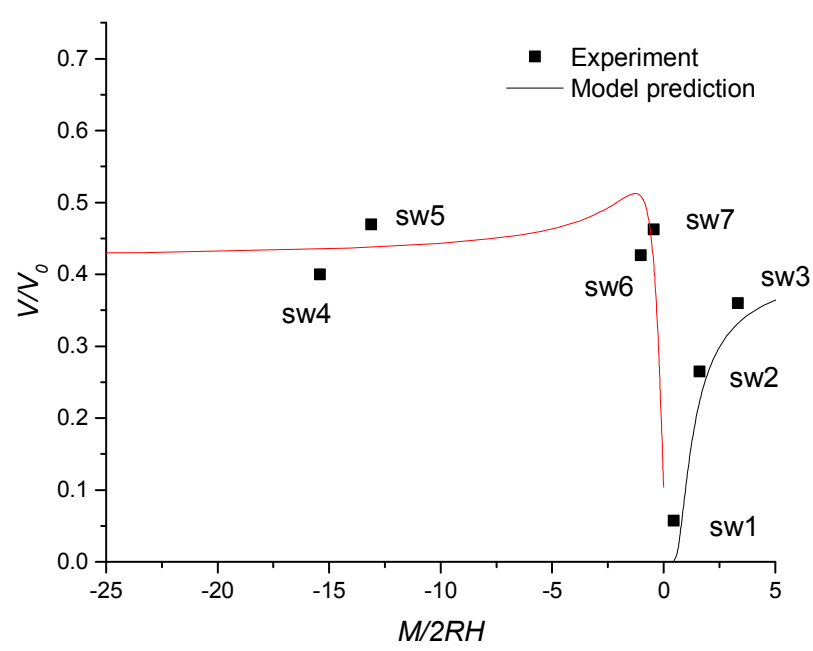

335 Fig. 12 Comparison between the experimental measured $V / V_{0}$ and $M / 2 R H$ values and model predictions

(a)

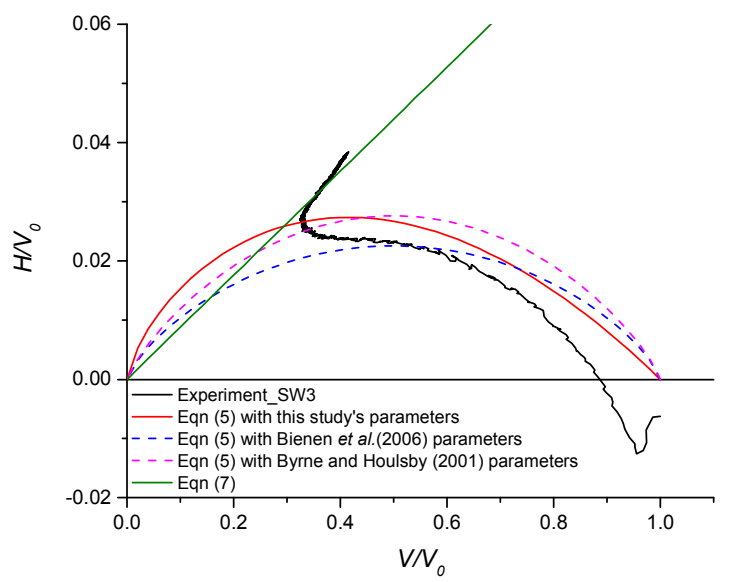

(b)

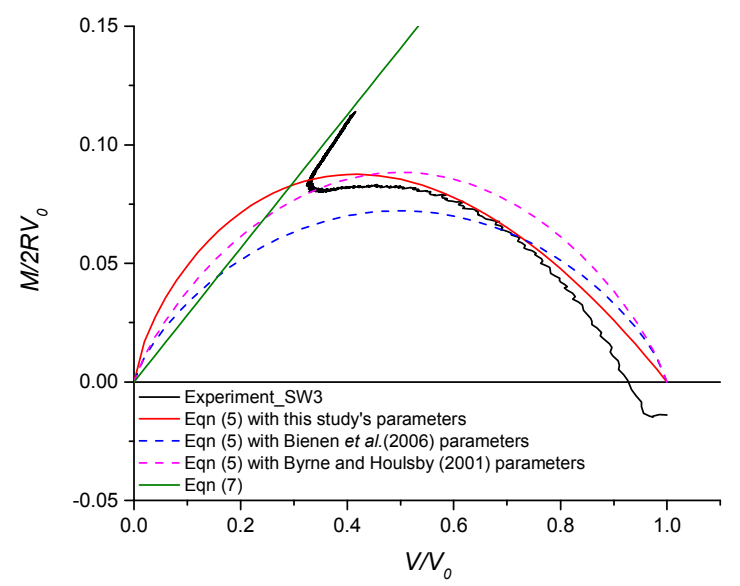

336

337

338

339

340

341

342

343

344

345

346

Fig. 13 Comparison between the experimental and theoretical yield surface and sliding surface

\section{Flow rule}

The prediction of footing displacement during yield requires a suitable flow rule. If the plastic flow vectors are perpendicular to the yield surface, then associate flow is assumed. However, Gottardi et al. (1999) found that for flat circular footings on sand the associated flow rule only models the ratios between plastic displacements in the $(H: M / 2 R)$ plane but not in the $(V: M)$ or $(V: H)$ planes. Associated flow rule under-predicts the level of the vertical displacements in these deviatoric planes, and this has been a consistent finding since, including in the experiments of Gottardi et al. (1999); Houlsby and Cassidy (2002), and Bienen et al. (2006). Houlsby and Cassidy (2002) and Cassidy et al. (2002a) defined a non-associated plastic potential based on the yield surface shape, but defined as

$g=\left(\frac{H}{\alpha_{h} h_{0} V_{0}{ }^{\prime}}\right)^{2}+\left(\frac{M / 2 R}{\alpha_{m} m_{0} V_{0}{ }^{\prime}}\right)^{2}-\frac{2 \alpha H M / 2 R}{\alpha_{h} \alpha_{m} h_{0} m_{0} V_{0}{ }^{\prime}}-\beta_{34}^{2}\left(\frac{V}{V_{0}{ }^{\prime}}\right)^{2 \beta_{3}}\left(1-\frac{V}{V_{0}{ }^{\prime}}\right)^{2 \beta_{4}}=0$ 
347 where

$348 \quad \beta_{34}=\frac{\left(\beta_{3}+\beta_{4}\right)^{\left(\beta_{3}+\beta_{4}\right)}}{\beta_{3}^{\beta 3} \beta_{4}^{\beta 4}}$

349 where $\alpha_{h}$ and $\alpha_{m}$ are non-dimensional horizontal and moment "association" factors, and $V_{0}$ ' is a 350 dummy parameter specifying the intersection of the plastic potential with the $V$-axis. The parameters $351 \beta_{3}$ and $\beta_{4}$ are chosen to allow for different variations in the curvature. If $\alpha_{h}, \alpha_{m}=1, \beta_{3}=\beta_{4}=1$, the 352 plastic potential coincides with the yield surface and an associated flow is implied. If $\alpha_{h}$ and $\alpha_{m}$ are 353 constant and equal, Equation (12) can be rewritten as

$g=\left(\frac{H}{h_{0} V_{0}{ }^{\prime}}\right)^{2}+\left(\frac{M / 2 R}{m_{0} V_{0}{ }^{\prime}}\right)^{2}-\frac{2 \alpha H M / 2 R}{h_{0} m_{0} V_{0}{ }^{\prime}}-\alpha_{v}{ }^{2} \beta_{34}{ }^{2}\left(\frac{V}{V_{0}{ }^{\prime}}\right)^{2 \beta_{3}}\left(1-\frac{V}{V_{0}{ }^{\prime}}\right)^{2 \beta_{4}}=0$

where $\alpha_{v}=\alpha_{h}=\alpha_{m}$

356 As two different governing equations exist for describing yield surface (Equation (5)) and sliding 357 surface (Equation (7)), the load paths that were tracing two surfaces are divided into two sections and 358 were therefore analysed separately. If the associated assumption applies, according to plasticity theory 359 the ratio of plastic displacement to rotation on yield surface and sliding surface can be evaluated as

360

${\frac{\delta u_{p}}{\delta \theta_{p} 2 R}}^{\text {(yield) }}=\frac{\partial f / \partial H}{\partial f / \partial(M / 2 R)}=\frac{H 2 R \frac{m_{0}}{h_{0}}-\alpha M}{M \frac{h_{0}}{m_{0}}-\alpha H 2 R}$

361 and

$362 \frac{\delta u_{p}}{\delta \theta_{p} 2 R}{ }^{\text {(liding) }}=\frac{\partial h / \partial H}{\partial h / \partial(M / 2 R)}=\frac{1}{-4 \tan \phi}$

(a)

(b) 

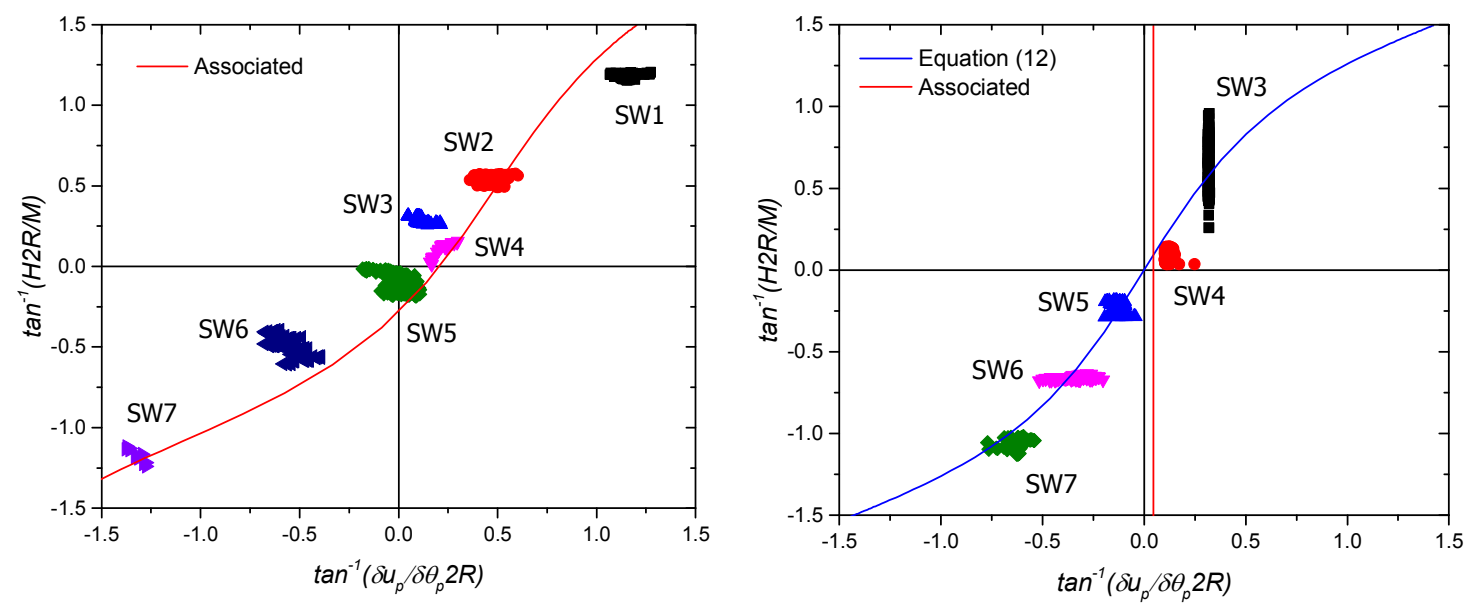

Fig. 14 Plastic flow on the $H: M$ plane for (a) yield surface and (b) sliding surface

364 Fig. 14(a) proves that associated flow is valid on yield surface in $H: M / 2 R$ plane by plotting the 365 experimental measured plastic displacement ratios against the analytical flow rule predictions. 366 However a non-associated behaviour was observed on sliding surface as showing in Fig. 14(b). For 367 the benefit of future modelling requirements, a plastic potential characterising the flow rule on the 368 sliding surface was proposed through a modification made to Equation (7) as

$j=\gamma \frac{1}{\tan \phi}\left(\frac{H}{V_{0}{ }^{\prime}}\right)^{2}-4 \lambda\left(\frac{M / 2 R}{V_{0}{ }^{\prime}}\right)^{2}+\frac{V}{V_{0}{ }^{\prime}}=0$

370 Similarly, $\lambda$ and $\gamma$ are the non-dimensional "association" factor, and $V_{0}^{\prime}$ ' is a dummy parameter. In 371 this study, the best fit non-dimensional association factors $\gamma$ and $\lambda$ were found to be $\tan \phi$ and -0.5 respectively. This is shown in Fig. 14(b).

373 On the $V: H$ and $V: M / 2 R$ planes, monotonic radial displacement tests were performed to evaluated 374 the flow rule. Further analysis of the experimental indicates that the associated assumption was not 375 supported in the deviatoric planes, therefore, Equation (9) can be further derived that

$\frac{\partial g}{\partial V}=\left(\alpha_{v} \beta_{34}\right)^{\frac{1}{\beta_{34}}}\left(\frac{V}{V_{0}^{\prime}}\right)^{\left(\frac{\beta_{3}}{\beta_{4}}-1\right)}\left(\frac{V}{V_{0}^{\prime}}-\frac{\beta_{3}}{\beta_{4}}\left(1-\frac{V}{V_{0}{ }^{\prime}}\right)\right) / V_{0}^{\prime}$

$$
\frac{\partial g}{\partial H}=\frac{1}{\beta_{4}}\left(\left(\frac{Q^{\prime}}{V_{0}^{\prime}}\right)^{2}\right)^{\left(\frac{1}{2 \beta_{4}}-1\right)}\left(\frac{H}{h_{0} V_{0}^{\prime}}-\frac{e M / 2 R}{\alpha_{m} m_{0} V_{0}^{\prime}}\right) / h_{0} V_{0}{ }^{\prime}
$$

$$
\frac{\partial g}{\partial M / 2 R}=\frac{1}{\beta_{4}}\left(\left(\frac{Q^{\prime}}{V_{0}^{\prime}}\right)^{2}\right)^{\left(\frac{1}{2 \beta_{4}}-1\right)}\left(\frac{M / 2 R}{\alpha_{m} m_{0} V_{0}^{\prime}}-\frac{e H}{h_{0} V_{0}^{\prime}}\right) / \alpha_{m} m_{0} V_{0}{ }^{\prime}
$$




$$
\frac{\delta q_{p}}{\delta w_{p}}=\frac{\partial g / \partial Q}{\partial g / \partial V}=\left(Q^{\prime} / V_{0}{ }^{\prime}\right) /\left(\alpha_{\mathrm{v}} \beta_{34}{ }^{2}\right)\left(1-\frac{V}{V_{0}{ }^{\prime}}\right)^{2 \beta_{4}}\left(\frac{V}{V_{0}{ }^{\prime}}\right)^{2 \beta_{3}}\left(\frac{\beta_{4}}{1-\frac{V}{V_{0}{ }^{\prime}}}-\frac{\beta_{3}}{\frac{V}{V_{0}^{\prime}}}\right)
$$

$380 \quad \beta_{34}=\frac{\left(\beta_{3}+\beta_{4}\right)^{\left(\beta_{3}+\beta_{4}\right)}}{\beta_{3}^{\beta_{3}} \beta_{4}^{\beta_{4}}}$

381 where $Q^{\prime}=\sqrt{\left(\frac{H}{\alpha_{h} h_{0}}\right)^{2}+\left(\frac{M / 2 R}{\alpha_{m} m_{0}}\right)^{2}-\frac{2 \alpha H M / 2 R}{\alpha_{h} \alpha_{m} h_{0} m_{0}}}$

382 Therefore, $\delta q_{p} / \delta w_{p}$ can be calculated. $\delta q_{p}$ is the plastic displacement in the general deviator plane, 383 as defined in Equation (6). An optimization scheme was adopted here to numerically adjust the values 384 of $\alpha_{v}, \beta_{3}$ and $\beta_{4}$ so that the difference between the predicted $\delta q_{p} / \delta w_{p}$ values and the experimental 385 measured $\delta q_{p} / \delta w_{p}$ values at a given $Q / V$ ratio was minimised. This is demonstrated in Fig. 15 as 386 the proposed non-associated flow rule represents most of the experimental data. The following values 387 were regarded as the best fit in this study: $\alpha_{v}=2.7, \beta_{3}=0.65, \beta_{4}=0.65$.
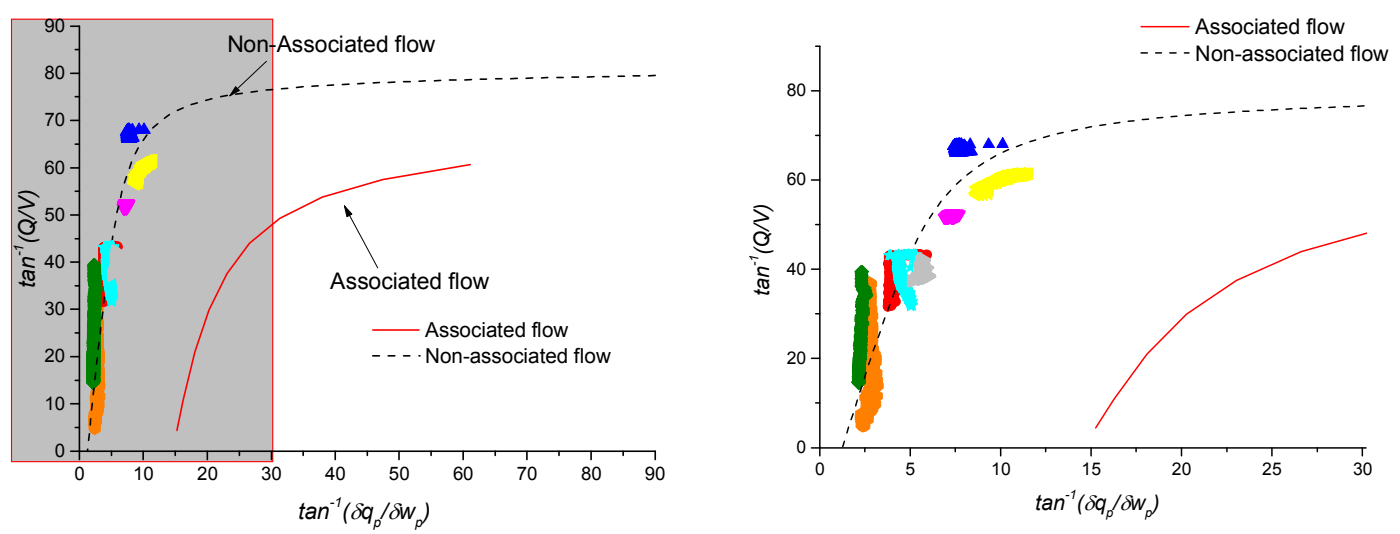

Fig. 15 Associated and best fit non-associated flow against the measured experimental data 


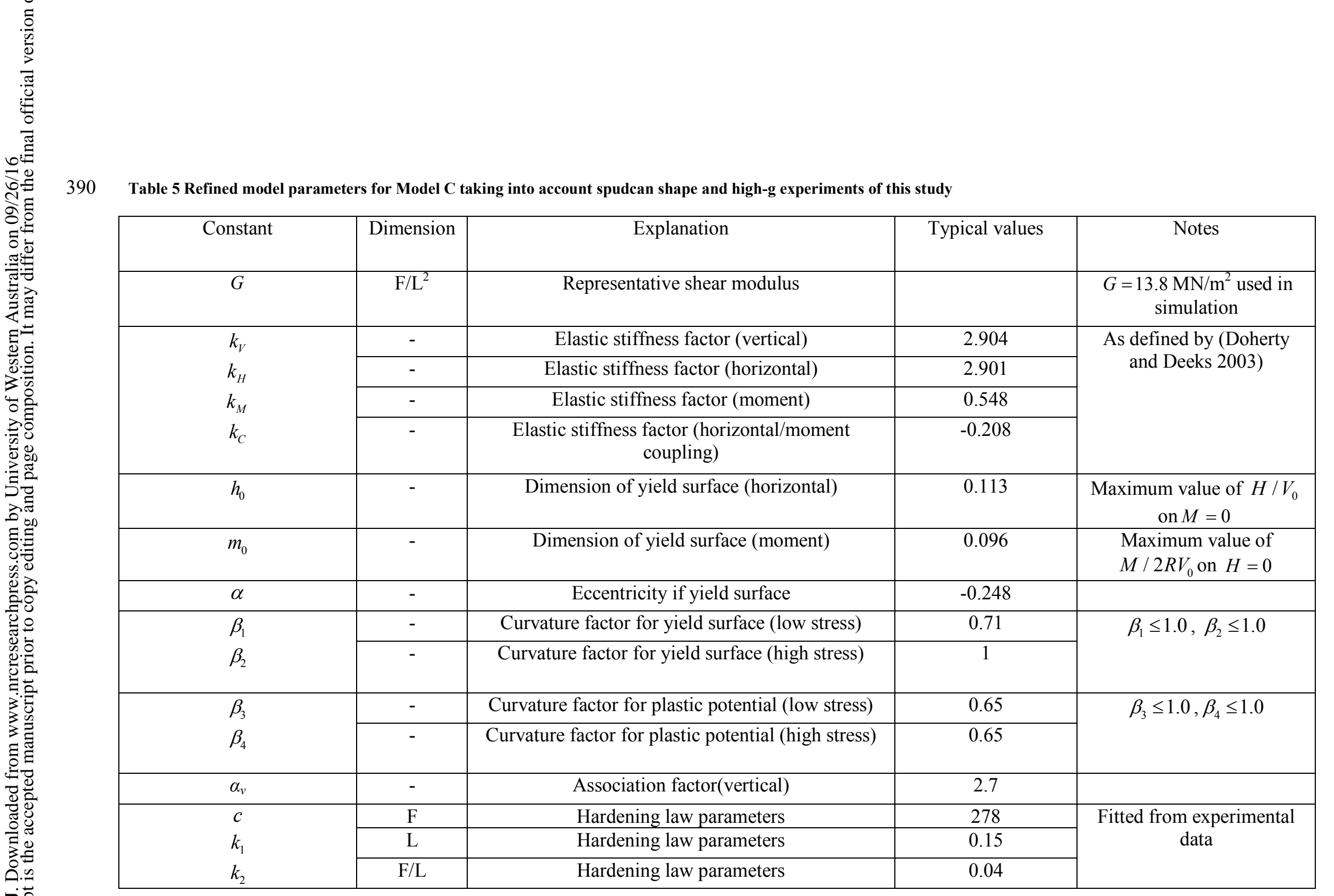




\section{Retrospective analyses}

392 The plasticity model of this study has been implemented into a FORTRAN programme to 393 demonstrate that the refined Model $C$ can be numerically implemented and used to simulate footing 394 behaviours. To investigate the capacity of this programme to predict footing behaviour, numerical 395 back analysis was carried out for a number of representative experiments.

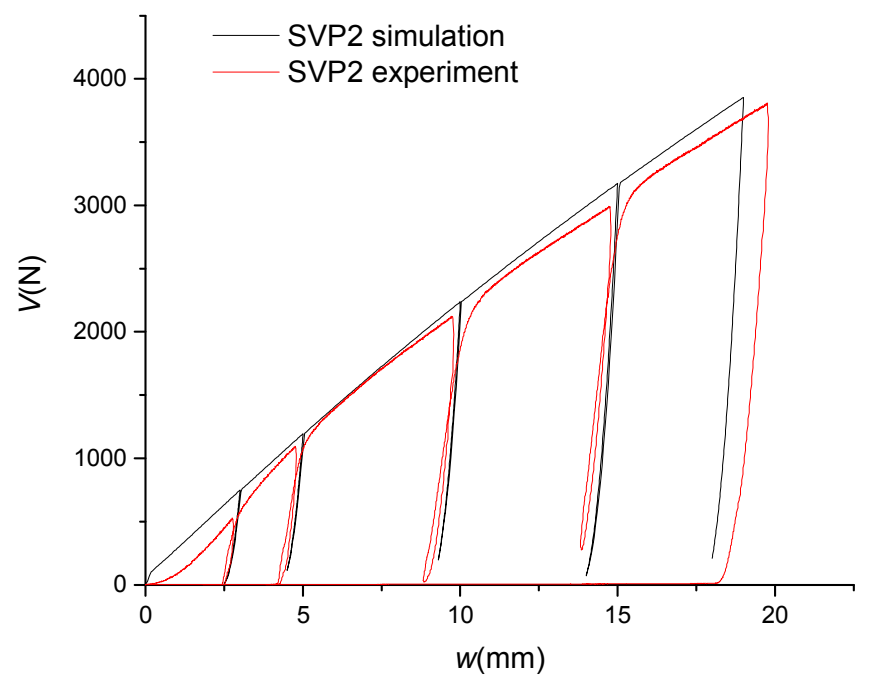

397

Fig. 16 Comparison between experimental data from test SVP2 and programme prediction

398 Fig. 16 shows the experimental results for a vertical load-unload loop test, SVP2, compared with a 399 simulation of this test in which the measured displacement is taken as input, and the vertical load is 400 calculated. Model $C$ with refined parameters produces a load that accurately represents the original 401 test, indicating that the chosen strain-hardening law and elastic stiffness are suitable. 

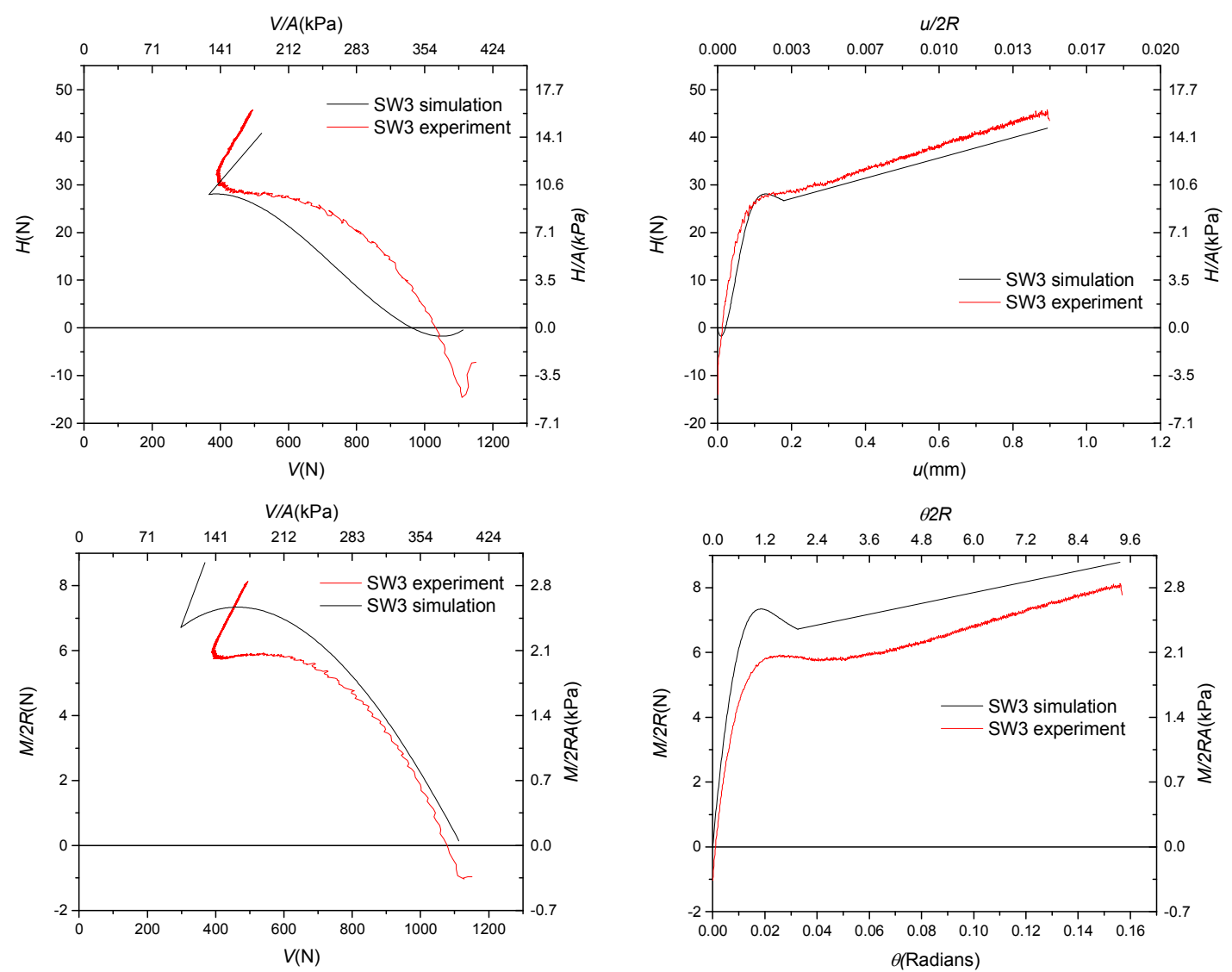

403

Fig. 17 Comparison between the experimental test, SW3, and the programme prediction

404 Fig. 17 represents a combined swipe test with $u / 2 R \theta=0.095$ starting at $V \approx 1100 N$ (SW3). The results are plotted in both the $V: H$ and the $V: M$ planes. In the numerical simulation, Model $C$ is load-controlled to $V \approx 1100 N$ and then displacement-controlled for the swipe. Model $C$ simulates the magnitude of the peak horizontal load adequately, reaching a value $H=28 \mathrm{~N}$, but the peak moment is slightly overestimated. Model $C$ locates the 'parallel point' perfectly in the $V: H$ plane; however, it again over- estimates in the $V: M$ plane. The difference between the experimental tests and simulation results in the early stage of the swipe test is due to a significant component of deviatoric load developed during the vertical loading phase of the swipe tests. This deviatoric load is due to the nature of displacement controlled test. Similar results can also be found in other studies, such as Zhang et al. (2013). 

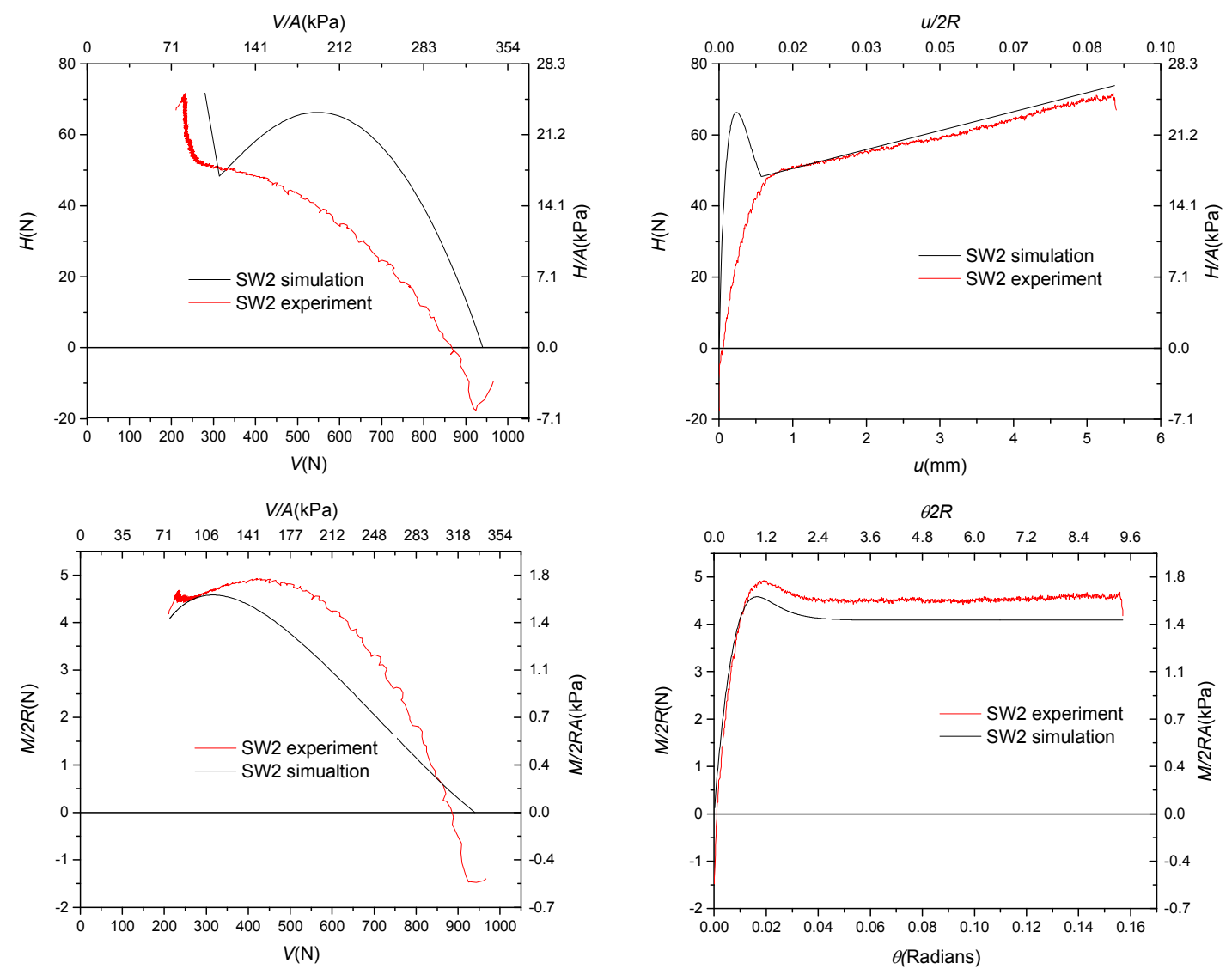

415

Fig. 18 Comparison between the experimental test, SW2, and the programme prediction

416 Fig. 18 represents the swipe test, SW2 with $u / 2 R \theta=0.531$ starting at $V \approx 950 N$. Model $C$ 417 overestimates the horizontal load but the simulation stops tracking at approximately the same 418 horizontal and vertical load, indicating an accurate prediction of the 'parallel point' in the horizontal 419 plane. For the equivalent test in the $V: M$ plane, the refined Model $C$ slightly underestimates the peak 420 moment but locates the 'parallel point' perfectly well. Further evidence that supports the model's 421 performance are also provided in Fig. 19 and Fig. 20 for tests SW1 and SW7 respectively. 

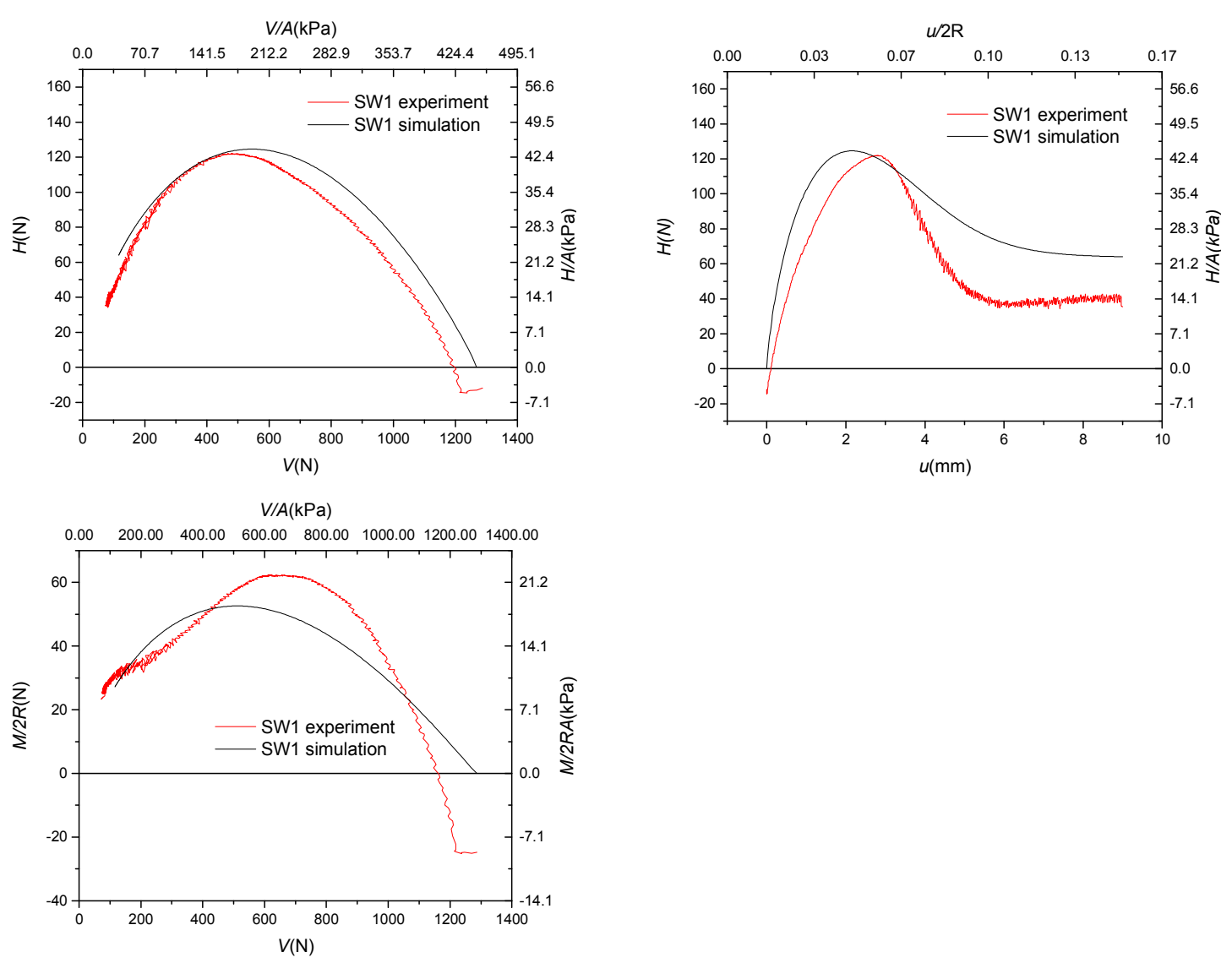

423

Fig. 19 Comparison between the experimental test, SW1, and the programme prediction 

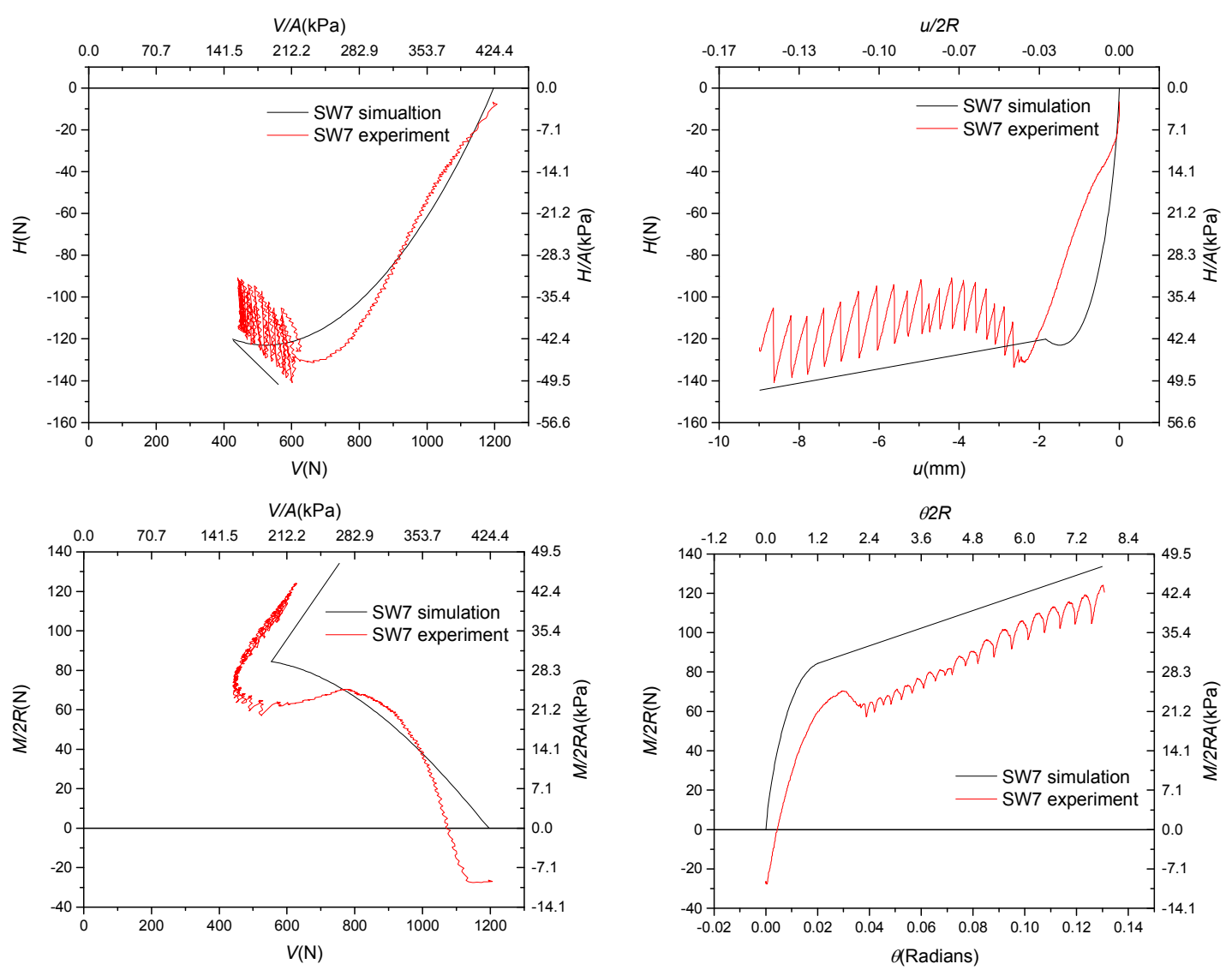

425

Fig. 20 Comparison between the experimental test, SW7, and the programme prediction

426 Constant gradients of the horizontal to vertical and the moment to vertical displacements were used as inputs to simulate the horizontal and moment radial displacement tests. The refined Model $C$ 428 predictions and the corresponding experimental horizontal and vertical loads are shown in Fig. 21. 429 The simulation is of a similar gradient, implying that the flow rule of modified Model $C$ is 430 performing well. 


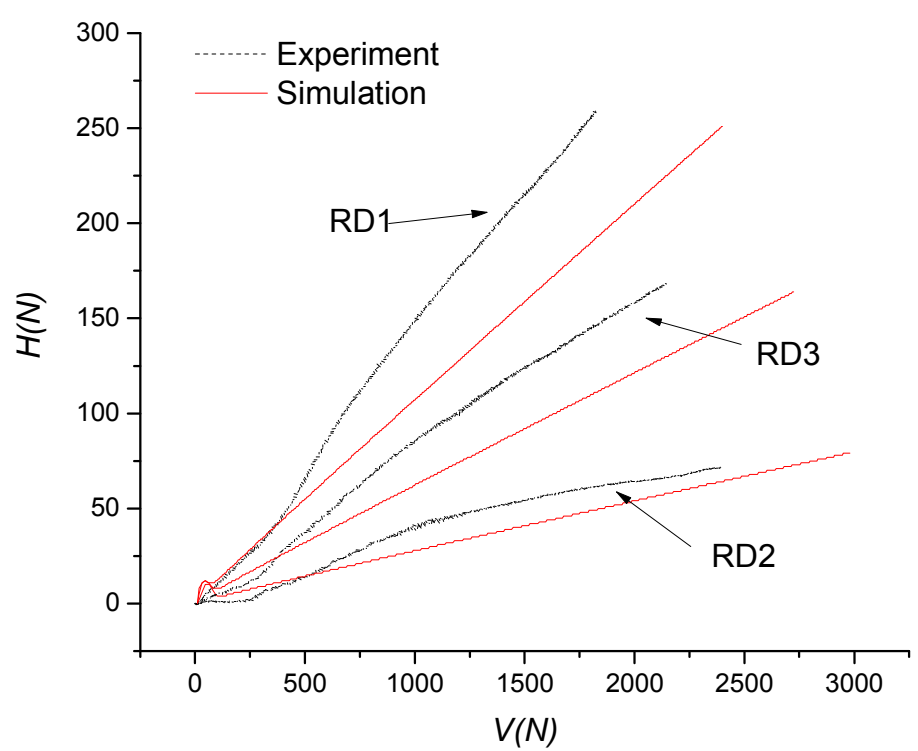

Fig. 21 Comparison between the experimental test RD1, RD2, RD3 and the programme prediction

\section{Conclusions}

434 In this paper, experimental results for an extensive research programme carried out in a drum 435 centrifuge at 100- $g$ are presented and interpreted within the strain-hardening plasticity framework. A three-dimensional sliding surface was observed in the experiments, and an analytical equation was subsequently proposed to describe this sliding surface. Comparison between this new yield-sliding analytical model and the experimental data demonstrates good agreement. The well-established threedimensional strain-hardening plasticity model (Model $C$ ) that can be used to describe flat circular footings has been experimentally recalibrated and refined for a spudcan footing resting on loose sand. Plasticity analysis shows that in the $H: M / 2 R$ plane, associated flow is valid for the yield surface, but non-associated flows were observed on the sliding surface. Consequently, a non-associated equation was proposed for the flow rule to capture this behaviour. Back-analysis of the experimental observations showed that the force-resultant model adequately captures the main features of the spudcan footing's behaviour although it remains conservative. The outcome from this study reveals the behaviour of a spudcan footing subject to combined loadings on loose sand under stress levels relevant to the offshore industry. The development of a complete strain-hardening plasticity model based on centrifuge observations has been achieved, adding further evidence to a well-established and widely used framework. This numerical model can be coupled with existing structural model thus an integrated analysis of structure-soil interactions can be performed. Further, a new sliding surface was observed in the tests and therefore added into the framework which completes the theory in describing footing's behaviour on frictional material under combined loadings. The need to model the load/displacement path of footing beyond the transition point reveals footing's true behaviour on 
454 frictional material under combined loadings. Without recognition of this sliding failure mechanism 455 leads to unconservative assumptions in practice. In response, this study provides greater confidence in 456 the sued of strain-hardening plasticity models in the assessment of jack-up platforms.

\section{Acknowledgements}

458 The first author acknowledges the support of the Australian Postgraduate Awards. The second author 459 acknowledges the support of the ARC through his Australian Laureate Fellowship (FL130100059) 460 and to the Lloyd's Register Foundation (LRF). LRF, a UK registered charity and sole shareholder of 461 Lloyd's Register Group Ltd, invests in science, engineering and technology for public benefit, 462 worldwide. The authors thank the following colleagues and technical staff at the Centre for Offshore 463 Foundation Systems at the University of Western Australia who provided invaluable support during 464 the instrumentation of the centrifuge test: Mr. Shane De Catania, Mr. Bart Thompson, Dr. Youhu 465 Zhang, and Dr. Conleth O’Loughlin. 


\section{References}

Bell, R. 1991. The analysis of offshore foundations subject to combined loading. University of Oxford.

Bienen, B., Byrne, B.W., Houlsby, G.T., and Cassidy, M.J. 2006. Investigating six-degree-of-freedom

loading of shallow foundations on sand. Géotechnique 56(6): 367-380.

Bienen, B., and Cassidy, M.J. 2006. Advances in the three-dimensional fluid-structure-soil interaction analysis of offshore jack-up structures. Marine Structures 19(2): 110-140. doi: DOI: 10.1016/j.marstruc.2006.09.002.

Bienen, B., and Cassidy, M.J. 2009. Three-dimensional numerical analysis of centrifuge experiments $\checkmark$ on a model jack-up drilling rig on sand. Canadian Geotechnical Journal 46(2): 208-224.

Bienen, B., Gaudin, C., and Cassidy, M.J. 2007. Centrifuge tests of shallow footing behaviour on sand under combined vertical-torsional loading. International Journal of Physical Modelling in Geotechnics 7(2): $1-21$.

Bienen, B., Gaudin, C., Cassidy, M.J., Rausch, L., and Purwana, O.A. 2012. Numerical modelling of undrained capacity of hybrid skirted foundation under combined loading. International Journal of Offshore and Polar Engineering 22(4): 323-329.

Butterfield, R., Houlsby, G.T., and Gottardi, G. 1997. Standardized sign conventions and notation for generally loaded foundations. Géotechnique 47(5): 1051-1054.

Byrne, B.W., and Houlsby, G.T. 1999. Drained behaviour of suction caisson foundations on very dense sand. In Offshore Technology Conference. Offshore Technology Conference, Houston, TX. pp. 3-6.

Byrne, B.W., and Houlsby, G.T. 2001. Observations of footing behaviour on loose carbonate sands. Géotechnique 51(5): 463-466.

Cassidy, M., and Cheong, J. 2005. The behaviour of circular footings on sand subjected to combined vertical-torsion loading. International Journal of Physical Modelling in Geotechnics 5(4): 1-14.

Cassidy, M.J. 2007. Experimental observations of the combined loading behaviour of circular

$\checkmark$ footings on loose silica sand. Geotechnique 57(4): 397-401.

Cassidy, M.J., Byrne, B.W., and Houlsby, G.T. 2002a. Modelling the behaviour of circular footings vunder combined loading on loose carbonate sand. Géotechnique 52(10): 705-712.

Cassidy, M.J., Byrne, B.W., and Randolph, M.F. 2004. A comparison of the combined load behaviour $\checkmark$ of spudcan and caisson foundations on soft normally consolidated clay. Géotechnique 54(2): 91-106.

Cassidy, M.J., Houlsby, G.T., Hoyle, M., and Marcom, M.R. 2002b. Determining appropriate stiffness levels for spudcan foundations using jack-up case records. In 21st International Conference on Offshore Mechanics and Arctic Engineering. American Society of Mechanical Engineers, Oslo, Norway. pp. 307-318.

Cassidy, M.J., Vlahos, G., and Hodder, M. 2010. Assessing appropriate stiffness levels for spudcan $\checkmark$ foundations on dense sand. Marine Structures 23(2): 187-208. doi: DOI: 10.1016/j.marstruc.2010.03.003.

Cheng, N., and Cassidy, M.J. 2016. Combined loading capacity of spudcan footings on loose sand. International Journal of Physical Modelling in Geotechnics 16(1): 31-44. 
Cheng, N., Gaudin, C., Cassidy, M.J., and Bienen, B. 2014. Centrifuge study of the combined bearing capacity of a hybrid foundation system. In 8th International Conference on Physical Modelling in Geotechnics, Perth. pp. 487-492.

Cocjin, M., and Kusakabe, O. 2012. Centrifuge observations on combined loading of a strip footing on dense sand. Géotechnique 63(5): 427-433.

Doherty, J.P., and Deeks, A.J. 2003. Elastic response of circular footings embedded in a nonhomogeneous half-space. Géotechnique 53(8): 703-714.

Gottardi, G., and Butterfield, R. 1993. On the bearing capacity of surface footings on sand under general planar loads. Soils and Foundations 33(3): 68-79.

Gottardi, G., and Butterfield, R. 1995. The displacement of a model rigid surface footing on dense sand under general planar loading. Soils and Foundations 35(3): 71-82.

Gottardi, G., Houlsby, G.T., and Butterfield, R. 1999. Plastic response of circular footings on sand -under general planar loading. Géotechnique 49(4): 453-469.

Govoni, L., Gourvenec, S., and Gottardi, G. 2010. Centrifuge modelling of circular shallow foundations on sand. International Journal of Physical Modelling in Geotechnics 10(2): 35-46.

Govoni, L., Gourvenec, S., and Gottardi, G. 2011. A centrifuge study on the effect of embedment on the drained response of shallow foundations under combined loading. Géotechnique 61(12): 10551068.

Hansen, J.B. 1970. A revised and extended formula for bearing capacity. Danish Geotechnical Institute, Copenhagen. pp. 5-11.

Houlsby, G.T., and Cassidy, M.J. 2002. A plasticity model for the behaviour of footings on sand under combined loading. Géotechnique 52(2): 117-129.

ISO. 2012. Petroleum and natural gas industries-Site specific assessment of mobile offshore units Part 1:Jack-ups, 19905-1. International Organization for Standardization.

Martin, C.M., and Houlsby, G.T. 2000. Combined loading of spudcan foundations on clay: laboratory tests. Géotechnique 50(4): 325-338.

Martin, C.M., and Houlsby, G.T. 2001. Combined loading of spudcan foundations on clay: numerical -modelling. Géotechnique 51(8): 687-699.

Meyerhof, G.G. 1953. The bearing capacity of foundations under eccentric and inclined loads. In 3rd International Conference on Soil Mechanics and Foundation Engineering, Switzerland. pp. 440-445.

National Instrument Corporation. 2003. LabVIEW User Manual. National Instrument Corporation, Austin,TX,USA.

Ngo-Tran, C. 1996. The analysis of offshore foundations subjected to combined loading. University of Oxford.

Noble Denton \& Associates. 1987. Foundation fixity of jack-up units: a joint industry study. London: Noble and Denton Associates.

Nova, R., and Montrasio, L. 1991. Settlements of shallow foundations on sand. Géotechnique 41(2): 243-256. 
Osborne, J.J., Trickey, J.C., Houlsby, G.T., and James, R.G. 1991. Findings from a joint industry study on foundation fixity of jackup units. In Offshore Technology Conference. Offshore Technology Conference, Houston, TX. pp. 517-533.

Schneider, J.A., and Lehane, B.M. 2006. Effects of width for square centrifuge displacement piles in sand. In Proceedings of the 6th International Conference on Physical Modelling in Geotechnics, Hong Kong. pp. 867-873.

Schotman, G.J.M. 1989. The effects of displacements on the stability of jackup spud-can foundations. In Offshore Technology Conference, Houston, TX. pp. 515-524.

Stewart, D., Boyle, R., and Randolph, M.F. 1998. Experience with a new drum centrifuge. In International conference centrifuge, Tokyo. pp. 35-40.

Tan, F.S.C. 1990. Centrifuge and theoretical modelling of conical footings on sand. University of Cambridge, Cambridge, UK.

Terzaghi, K. 1943. Theoretical soil mechanics. John Wiley and Sons.

White, D.J., Teh, K.L., Leung, C.F., and Chow, Y.K. 2008. A comparison of the bearing capacity of - flat and conical circular foundations on sand. Géotechnique 58(10): 781-792.

Zhang, Y., Bienen, B., and Cassidy, M.J. 2013. Development of a combined VHM loading apparatus for a geotechnical drum centrifuge. International Journal of Physical Modelling in Geotechnics 13(1): $13-30$.

Zhang, Y., Bienen, B., Cassidy, M.J., and Gourvenec, S. 2011. The undrained bearing capacity of a - spudcan foundation under combined loading in soft clay. Marine Structures 24(4): 459-477. doi: http://dx.doi.org/10.1016/j.marstruc.2011.06.002. 